\title{
GEOMETRIC CHAIN HOMOTOPY EQUIVALENCES BETWEEN NOVIKOV COMPLEXES
}

\author{
D. SCHÜTZ
}

\begin{abstract}
We give a detailed account of the Novikov complex corresponding to a closed 1 -form $\omega$ on a closed connected smooth manifold $M$. Furthermore we deduce the simple chain homotopy type of this complex using various geometrically defined chain homotopy equivalences and show how they are related to another.
\end{abstract}

\section{INTRODUCTION}

The purpose of this article is to give a detailed exposition of the Novikov complex as defined in Novikov [11], and its chain homotopy type. Given a closed 1-form $\omega$ on a closed connected smooth manifold $M$ with only nondegenerate critical points, this complex is freely generated by the critical points over an appropriate ring. The grading of the complex is given by the indices of the critical points. To define the boundary operator a vector field $v$ gradient to $\omega$ is required. The boundary is then given by counting the trajectories between critical points.

In the case where $\omega$ is exact this complex has already been described by Milnor $[9, \S 7]$ and the ring can be chosen to be $\mathbb{Z}$ or a group ring $\mathbb{Z} G$, if a regular covering $\rho: \tilde{M} \rightarrow M$ with covering transformation group $G$ is considered. But in the nonexact case the group ring $\mathbb{Z} G$ no longer works and we have to use a completion $\widehat{\mathbb{Z}}_{\xi}$ of $\mathbb{Z} G$. Now $G$ is the covering transformation group of a regular covering $\rho: \tilde{M} \rightarrow M$ such that $\omega$ pulls back to an exact form. The completion also depends on a homomorphism $\xi: G \rightarrow \mathbb{R}$ induced by $\omega$.

As it turns out, the Novikov complex $C_{*}(\tilde{M}, \omega, v)$ is chain homotopy equivalent to $\widehat{\mathbb{Z}}_{\xi} \otimes_{\mathbb{Z} G} C_{*}(\tilde{M})$, where $C_{*}(\tilde{M})$ is the singular chain complex of $\tilde{M}$. Even though a first version was already announced in [11], detailed proofs did not appear until much later, see Latour [8] or Pajitnov [12]. Since then easier proofs have appeared based on geometrically defined chain homotopy equivalences. These equivalences appear in various places in the literature, but are not very well connected to each other. Given a smooth triangulation $\Delta$ of $M$, there is a chain homotopy equivalence $\varphi_{v}: \widehat{\mathbb{Z} G}_{\xi} \otimes_{\mathbb{Z} G} C_{*}^{\Delta}(\tilde{M}) \rightarrow C_{*}(\tilde{M}, \omega, v)$ based on intersection numbers between simplices and unstable manifolds of critical points. Versions of this equivalence appeared in Hutchings and Lee [7] and Schwarz [24], and the torsion properties have been discussed in [22].

1991 Mathematics Subject Classification. Primary 57R70; Secondary 55U15.

Key words and phrases. Morse-Smale complex, Novikov complex.

The author was partially supported by the EU under the TMR network FMRX CT97-0107 "Algebraic K-Theory, Linear Algebraic Groups and Related Structures" and by a Forschungsstipendium of the Max-Planck-Gesellschaft. 
Given another closed 1-form $\omega^{\prime}$ cohomologous to $\omega$ with a gradient $w$ there are different ways to describe chain homotopy equivalences $\psi_{v, w}: C_{*}(\tilde{M}, \omega, v) \rightarrow$ $C_{*}\left(\tilde{M}, \omega^{\prime}, w\right)$, see, for example, Latour $[8, \S 2.21]$ or Poźniak $[19, \S 2.6]$. We show that up to chain homotopy these definitions agree and that $\psi_{v, w}$ commutes with $\varphi_{v}$ and $\varphi_{w}$.

The techniques in this paper are based on Milnor [9]. In Section 2 we recall some of the results of [9] and show how to obtain $\varphi_{v}$ in the case of an ordinary Morse function. In Section 3 we look at the special case of a closed 1-form coming from a circle valued Morse function $f: M \rightarrow S^{1}$. It turns out that the results in the circle valued case can be reduced to the exact case by using inverse limit arguments. The general case of an arbitrary closed 1 -form is then reduced to the circle valued case in Section 4 using approximation arguments. Section 5 shows how the principle of continuation as described in Poźniak [19, §2.6] fits into our description. Then we discuss the simple chain homotopy type of the Novikov complex. For the basic notion of torsion and simple chain homotopy type we refer the reader to Cohen [1]. It turns out that the torsion of all discussed equivalences are represented by so called trivial units. It has been known that these torsions carry information on the closed orbit structure of the gradients in form of zeta functions. We recall these results in Section 7. Finally we give an example of a gradient where the noncommutative zeta function contains more information than the commutative zeta function.

A lot of the results in this paper were already collected in [23, App.A], but in a very sketchy form. Here we give complete proofs which are new in certain cases.

Describing the Novikov complex as an inverse limit was already done in Pajitnov [12]; Latour [8] and Poźniak [19] use quite a different approach. A more algebraic method to obtain the Novikov complex is used in Farber [2], Farber and Ranicki [3] and Ranicki [20], but the boundary operator there is no longer described by trajectories between critical points in general.

If $G$ is a group, we denote by $\mathbb{Z}^{G}$ the abelian group of all functions $\lambda: G \rightarrow \mathbb{Z}$. The group ring $\mathbb{Z} G \subset \mathbb{Z}^{G}$ consists of all $\lambda \in \mathbb{Z}^{G}$ such that $\lambda(g)=0$ for all but finitely many $g \in G$. For $g \in G$ we define $\lambda_{g} \in \mathbb{Z} G$ by $\lambda_{g}(h)=0$ for $h \neq g$ and $\lambda_{g}(g)=1$. By abuse of notation we write $g=\lambda_{g} \in \mathbb{Z} G$.

I am grateful to the Department of Mathematics and Statistics at the University of Edinburgh and to the Max-Planck-Institut für Mathematik at Bonn where parts of this paper were written. I would also like to thank Ross Geoghegan and Andrew Ranicki for suggestions and valuable discussions.

\section{The Morse-Smale COMPlex}

Let $\left(W ; M_{0}, M_{1}\right)$ be a compact cobordism between the closed manifolds $M_{0}$ and $M_{1}$. A Morse function $f$ on $W$ is a smooth function $f: W \rightarrow[0,1]$ such that $f^{-1}(\{0\})=M_{0}, f^{-1}(\{1\})=M_{1}$ and the critical points of $f$ are in the interior and nondegenerate. It follows that $f$ has only finitely many critical points and every such point $p \in W$ has an index denoted by ind $p$. The set of critical points of index $i$ will be denoted by $\operatorname{crit}_{i}(f)$. The existence of Morse functions is shown in Milnor $[9, \S 3]$.

Let $v$ be an $f$-gradient, i.e. a vector field dual to the exact 1 -form $d f$ with respect to some Riemannian metric. Let $\Phi$ be the flow of $v$. The stable and unstable 
manifolds at a critical point are defined as

$$
\begin{aligned}
W^{s}(p, v) & =\{x \in W \mid \Phi(x, t) \rightarrow p \text { for } t \rightarrow \infty\} \\
W^{u}(p, v) & =\{x \in W \mid \Phi(x, t) \rightarrow p \text { for } t \rightarrow-\infty\}
\end{aligned}
$$

Then $W^{s}(p, v)$ is an immersed submanifold of dimension ind $p$ and $W^{u}(p, v)$ one of dimension $n-$ ind $p$. If $W^{s}(p, v)$ and $W^{u}(q, v)$ intersect transversely for all critical points with ind $p<$ ind $q$, we can find a filtration $M_{0}=W_{-1} \subset W_{0} \subset \ldots \subset W_{n}=$ $W$ of $W$ by compact cobordisms such that $W_{i}-$ int $W_{i-1}$ is a compact cobordism that contains exactly the critical points of index $i$ in its interior. Furthermore if $x \in W_{i}$, so is $\Phi(x, t)$ for all $t<0$ such that $\Phi(x, t)$ is defined.

Define $C_{i}\left(W, M_{0}, f, v\right)=H_{i}\left(W_{i}, W_{i-1}\right)$ and $\partial: C_{i}\left(W, M_{0}, f, v\right) \rightarrow C_{i-1}\left(W, M_{0}, f, v\right)$ to be the connecting homomorphism of the triple $\left(W_{i}, W_{i-1}, W_{i-2}\right)$. Then $C_{i}(W$, $\left.M_{0}, f, v\right)$ is a free abelian group of rank $\left|\operatorname{crit}_{i}(f)\right|$ and the stable manifolds of the critical points represent the generators. Furthermore we have $H_{*}\left(C_{*}\left(W, M_{0}, f, v\right)\right)=$ $H_{*}\left(W, M_{0}\right)$.

If $p: \tilde{W} \rightarrow W$ is a regular covering space with covering transformation group $G$, define $\tilde{A}=p^{-1}(A)$ for subsets $A \subset W$. Then let $C_{i}\left(\tilde{W}, \tilde{M}_{0}, f, v\right)=H_{i}\left(\tilde{W}_{i}, \tilde{W}_{i-1}\right)$ and let the boundary be defined using the triple $\left(\tilde{W}_{i}, \tilde{W}_{i-1}, \tilde{W}_{i-2}\right)$. Then $C_{*}\left(\tilde{W}, \tilde{M}_{0}, f, v\right)$ is a finitely generated free chain complex over $\mathbb{Z} G$ generated by the critical points of $f$ and its homology is $H_{*}\left(\tilde{W}, \tilde{M}_{0}\right)$.

Notice that the chain groups are independent of the filtration. To express the boundary homomorphism in terms of the vector field alone, assume now also that $W^{s}(p, v)$ and $W^{u}(q, v)$ intersect transversely for all critical points of $f$ with ind $p \leq$ ind $q+1$. For ind $p=$ ind $q+1=i+1$ we get that $W^{s}(p, v) \cap W^{u}(q, v) \cap \partial W_{i}$ is a compact 0 -dimensional manifold. Therefore we have finitely many trajectories between $p$ and $q$.

Definition 2.1. An $f$-gradient $v$ is called transverse, if $W^{s}(p, v)$ and $W^{u}(q, v)$ intersect transversely for all critical points of $f$ with ind $p \leq$ ind $q+1$.

Choose an orientation for every stable manifold $W^{s}(p, v)$ and orient the normal bundle of $W^{u}(p, v)$ such that the orientation of the normal bundle of $W^{u}(p, v)$ at $p$ projects to the orientation of $W^{s}(p, v)$ at $p$. An orientation of the normal bundle will also be called a coorientation. Now let $\gamma$ be a trajectory of $-v$ between $p$ and $q$. For $t \in \mathbb{R}$ we have $d f_{\gamma(t)}\left(\gamma^{\prime}(t)\right)<0$. Let $X_{1}, \ldots, X_{i} \in T_{\gamma(t)} M$ represent the coorientation of $W^{u}(q, v)$. If the projection of $\gamma^{\prime}(t), X_{1}, \ldots, X_{i}$ into $T_{\gamma(t)} W^{s}(p, v)$ represents the orientation of $W^{s}(p, v)$, set $\varepsilon(\gamma)=1$, otherwise set $\varepsilon(\gamma)=-1$. Note that the projection does represent a basis of $T_{\gamma(t)} W^{s}(p, v)$ by the transversality assumption.

Now lift the orientations to $\tilde{W}$ and choose for every critical point $p$ of $f$ exactly one lift $\tilde{p}$ in $\tilde{W}$. For critical points $p, q$ with ind $p=\operatorname{ind} q+1$ define $[p: q] \in \mathbb{Z} G$ by

$$
[p: q](g)=\sum \varepsilon(\gamma)
$$

where the sum is taken over the finite set of trajectories between $\tilde{p}$ and $g \tilde{q}$. It turns out that $\partial: C_{i+1}\left(\tilde{W}, \tilde{M}_{0}, f, v\right) \rightarrow C_{i}\left(\tilde{W}, \tilde{M}_{0}, f, v\right)$ is given by

$$
\partial(p)=\sum_{q, \text { ind } q=i}[p: q] q
$$


when we identify $p$ with the homology class of $W^{s}(\tilde{p}, \tilde{v})$ in $H_{i+1}\left(\tilde{W}_{i+1}, \tilde{W}_{i}\right)$, see Milnor $[9, \S 7]$.

Definition 2.2. The Morse-Smale complex of the Morse function $f$ with transverse $f$-gradient $v$ is given by

$$
C_{i}^{M S}\left(\tilde{W}, \tilde{M}_{0}, f, v\right)=\bigoplus_{p \in \operatorname{crit}_{i}(f)} \mathbb{Z} G
$$

and the boundary is given by (1).

This gives an abstract definition of the Morse-Smale complex in terms of critical points and trajectories between them, but the description using relative homology groups will remain useful. Let us modify the filtrations for this.

Since $M_{0}$ and $M_{1}$ can be nonempty, the flow of $-v$ need not be defined on all of $W \times \mathbb{R}$, but we can use it to define a continuous map $\Theta: W \times \mathbb{R} \rightarrow W$ which agrees with the flow where the flow is defined. To do this we can put a collar on $W$ to get a slightly bigger manifold $W_{c}$ and extend $-v$ to $W_{c}$ such that the new vector field vanishes on $\partial W_{c}$. The flow of the new vector field is now defined on $W_{c} \times \mathbb{R}$ and we get $\Theta$ by retracting $W_{c}$ back to $W$. In particular we get $\Theta(x, t)=x$ for $(x, t) \in M_{0} \times[0, \infty) \cup M_{1} \times(-\infty, 0]$. For $t \in \mathbb{R}$ set $\Theta_{t}(x)=\Theta(x, t)$ and define

$$
W_{i}^{t}=\left\{\begin{array}{cc}
\Theta_{t}\left(W_{i}\right) & \text { if } t \geq 0 \\
\bigcup_{0 \geq s \geq t} \Theta_{s}\left(W_{i}\right) & \text { if } t<0
\end{array}\right.
$$

Then $\left(W_{i}^{t}\right)_{i=-1}^{n}$ is also a filtration and clearly $H_{i}\left(\tilde{W}_{i}^{t}, \tilde{W}_{i-1}^{t}\right) \cong H_{i}\left(\tilde{W}_{i}, \tilde{W}_{i-1}\right)$ where the isomorphism is induced by inclusion.

Note that for $t>s$ we have $W_{i}^{t} \subset W_{i}^{s}$ and for very large $t W_{i}^{t}$ mainly consists of $M_{0}$ and the stable manifolds of critical points with index $\leq i$. Also for very negative $s W_{i}^{s}$ is mainly $W$ minus the unstable manifolds of critical points with index $\geq i+1$. To make this more precise note that for $t>s$ we get a direct system $j_{t s}=j_{*}: H_{*}\left(\tilde{W}_{i}^{t}, \tilde{W}_{i-1}^{t}\right) \rightarrow H_{i}\left(\tilde{W}_{i}^{s}, \tilde{W}_{i-1}^{s}\right)$ consisting of isomorphisms and commuting diagrams

$$
\begin{array}{ccc}
H_{i}\left(\tilde{W}_{i}^{t}, \tilde{W}_{i-1}^{t}\right) & \stackrel{\partial_{i}}{\longrightarrow} & H_{i-1}\left(\tilde{W}_{i-1}^{t}, \tilde{W}_{i-2}^{t}\right) \\
\downarrow j_{t s} & & \downarrow j_{t s} \\
H_{i}\left(\tilde{W}_{i}^{s}, \tilde{W}_{i-1}^{s}\right) & \stackrel{\partial_{i}}{\longrightarrow} & H_{i-1}\left(\tilde{W}_{i-1}^{s}, \tilde{W}_{i-2}^{s}\right)
\end{array}
$$

Let us define

$$
C_{i}=C_{i}(v)=W-\bigcup_{p, \text { ind } p \geq i+1} W^{u}(p, v) .
$$

It is easy to see that $H_{i}\left(\tilde{C}_{i}, \tilde{C}_{i-1}\right)$ is the direct limit of the above direct system and since all $j_{t s}$ are isomorphisms we can describe the Morse-Smale complex in terms of this filtration.

Definition 2.3. Let $\Delta$ be a smooth triangulation of $W$ which contains $M_{0}$ and $M_{1}$ as subcomplexes. We say $\Delta$ is adjusted to $v$, if every $i$-simplex $\sigma^{i}$ intersects the unstable manifolds $W^{u}(p, v)$ transversely for all critical points $p$ with ind $p \geq i$.

To see the existence of adjusted triangulations, let $\psi: W \rightarrow W$ be a diffeomorphism homotopic to the identity and $\Delta$ a smooth triangulation of $W$. Then $\psi \Delta$ is the 
triangulation of $W$ where simplices are composed with $\psi$. Then the corresponding chain complexes can be identified by choosing a lifting $\tilde{\psi}: \tilde{W} \rightarrow \tilde{W}$. homotopic to the identity.

So let $\Delta$ be any smooth triangulation and $\psi_{-1}=\mathrm{id}_{W}$. We can adjust $\psi_{-1}$ near the 0 -skeleton so that 0 -simplices intersect all unstable manifolds transversely. Since the boundary of $W$ is transverse to the flow, we can leave it invariant. This way we get a diffeomorphism $\psi_{0}$ isotopic to the identity. Now assume $\psi_{k-1}$ is isotopic to the identity and every $j$-simplex of $\psi_{k-1} \Delta$ with $j \leq k-1$ intersects the unstable manifolds transversely for critical points with index $\geq k-1$. We modify $\psi_{k-1}$ on the $k$-skeleton so that $k$-simplices intersect $W^{u}(p, v)$ transversely for all $p$ with index $\geq k$. Notice that for a $k$-simplex of $\psi_{k-1} \Delta$ this is already true for $\psi_{k-1}$ near the boundary so we can leave the $(k-1)$-skeleton fixed. This way we obtain $\psi_{k}$ isotopic to the identity and we can proceed by induction.

Then $\psi_{n-1} \Delta$ is adjusted to $v$. Furthermore we can find an adjusted triangulation $\psi \Delta$ with $\psi$ as close as we like to the identity. Moreover, compactness gives that if $\Delta$ is adjusted to $v$, so is $\psi \Delta$ for every $\psi$ close enough to the identity.

Denote the $i$-skeleton of a triangulation by $W^{(i)}$. If $\Delta$ is adjusted to $v$, we get that $W^{(i)} \subset C_{i}$. In particular the inclusion induces a map on homology $j_{*}$ : $H_{*}\left(\tilde{W}^{(i)}, \tilde{W}^{(i-1)}\right) \rightarrow H_{*}\left(\tilde{C}_{i}, \tilde{C}_{i-1}\right)$ which defines a chain map

$$
\varphi_{v}: C_{*}^{\Delta}\left(\tilde{W}, \tilde{M}_{0}\right) \rightarrow C_{*}^{M S}\left(\tilde{W}, \tilde{M}_{0}, f, v\right) .
$$

If ind $p=\operatorname{dim} \sigma$, we have that $\sigma \cap W^{u}(p, v)$ is a finite set by the transversality assumption. For the definition of $C_{*}^{\Delta}\left(\tilde{W}, \tilde{M}_{0}\right)$ as generated by simplices, every simplex has a chosen lift and an orientation. Using the coorientation of $W^{u}(p, v)$ we get an intersection number $[\sigma: p] \in \mathbb{Z} G$ such that

$$
\varphi_{v}(\sigma)=\sum_{p, \operatorname{ind} p=\operatorname{dim} \sigma}[\sigma: p] p
$$

so $\varphi_{v}$ can be expressed in terms of $v$ only.

If we are given an arbitrary smooth triangulation of the cobordism $W$, the above construction shows how to modify the triangulation to get a chain map. Different maps $\psi_{1}, \psi_{2}$ can lead to different chain maps, but the chain homotopy type is well defined:

Lemma 2.4. The chain maps induced by $\psi_{1} \Delta$ and $\psi_{2} \Delta$ are chain homotopic.

Proof. Let $H^{\prime}: W \times I \rightarrow W$ be a homotopy between $\psi_{1}$ and $\psi_{2}$. As above we can change $H^{\prime}$ to a homotopy $H: W \times I \rightarrow W$ between $\psi_{1}$ and $\psi_{2}$ such that $H(\sigma \times I)$ intersects $W^{u}(p, v)$ transversely for all critical points $p$ with ind $p \geq \operatorname{dim} \sigma+1$. Lift $H$ to a homotopy $\tilde{H}: \tilde{W} \times I \rightarrow \tilde{W}$ such that $\tilde{H}_{0}=\tilde{\psi}_{1}$ is homotopic to the identity. Then define $H_{i}: C_{i}^{\Delta}\left(\tilde{W}, \tilde{M}_{0}\right) \rightarrow C_{i+1}^{M S}\left(\tilde{W}, \tilde{M}_{0}, f, v\right)$ by $H_{i}(\sigma)=(-1)^{i} \tilde{H}_{*}[\tilde{\sigma} \times I] \in$ $H_{i+1}\left(C_{i+1}, C_{i}\right)$. Then

$$
\begin{aligned}
\partial H+H \partial(\sigma) & =(-1)^{i} \tilde{H}_{*} \partial[\tilde{\sigma} \times I]+(-1)^{i-1} \tilde{H}_{*}[\partial \tilde{\sigma} \times I] \\
& =\tilde{H}_{*}[\tilde{\sigma} \times 1]-\tilde{H}_{*}[\tilde{\sigma} \times 0] \\
& =\tilde{\psi}_{2}[\tilde{\sigma}]-\tilde{\psi}_{1}[\tilde{\sigma}]
\end{aligned}
$$

so $H_{i}$ is the desired chain homotopy. 
We already know that the triangulated and the Morse-Smale complex have the same homology and now we show that $\varphi_{v}$ is indeed a chain homotopy equivalence.

Theorem 2.5. Let $f: W \rightarrow[a, b]$ be a Morse function, $v$ a transverse $f$-gradient, $\Delta$ a triangulation adjusted to $v$ and $p: \tilde{W} \rightarrow W$ a regular covering space. Then $\varphi_{v}: C_{*}^{\Delta}\left(\tilde{W}, \tilde{M}_{0}\right) \rightarrow C_{*}^{M S}\left(\tilde{W}, \tilde{M}_{0}, f, v\right)$ is a simple homotopy equivalence.

Proof. Let $\Delta^{\prime}$ be a subdivision of $\Delta$. If $\psi \Delta^{\prime}$ is adjusted to $v$, so is $\psi \Delta$. Moreover, the diagram

$$
\begin{array}{cc}
C_{*}^{\psi \Delta}\left(\tilde{W}, \tilde{M}_{0}\right) \stackrel{\text { sd }}{\longrightarrow} & C_{*}^{\psi \Delta^{\prime}}\left(\tilde{W}, \tilde{M}_{0}\right) \\
\varphi_{v} \searrow & \swarrow \varphi_{v} \\
C_{*}^{M S}\left(\tilde{W}, \tilde{M}_{0}\right)
\end{array}
$$

commutes, where sd is subdivision, a simple homotopy equivalence. By Munkres $[10, \S 10]$ it is good enough to show the theorem for a special smooth triangulation. Recall the filtration $\left(W_{i}\right)_{i=-1}^{n}$ by compact cobordisms. Choose a triangulation such that each $W_{i}$ is a subcomplex for all $-1 \leq i \leq n$ and so that for each critical point $p$ of index $i$ the disc $D_{i}(p)=W^{s}(p, v) \cap\left(W_{i}-\operatorname{int} W_{i-1}\right)$ is a subcomplex. We set for $0 \leq k \leq n C_{*}^{(k)}=C_{*}^{\Delta}\left(\tilde{W}_{k}, \tilde{M}_{0}\right)$. The complex $D_{*}^{(k)}$ is given by

$$
D_{i}^{(k)}=\left\{\begin{array}{cl}
C_{i}^{M S}\left(\tilde{W}, \tilde{M}_{0}\right) & i \leq k \\
0 & \text { otherwise }
\end{array}\right.
$$

The chain map $\varphi_{v}$ induces maps $\varphi^{(k)}: C_{*}^{(k)} \rightarrow D_{*}^{(k)}$ and $\varphi^{(k, k-1)}: C_{*}^{(k)} / C_{*}^{(k-1)} \rightarrow$ $D_{*}^{(k)} / D_{*}^{(k-1)}$. Since the diagram

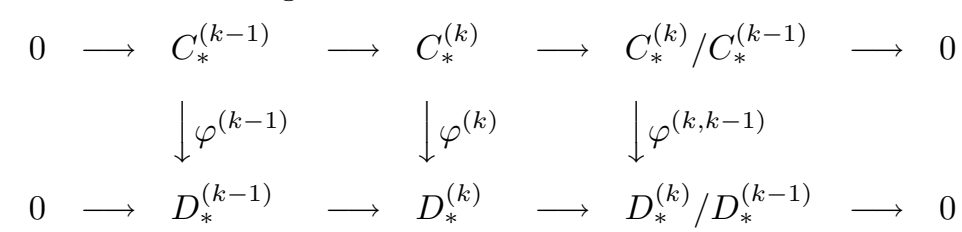

commutes, it suffices to show that each $\varphi^{(k, k-1)}$ is a simple homotopy equivalence to finish the proof.

Clearly $\varphi^{(k, k-1)}$ induces an isomorphism in homology, so it remains to show that it is simple. We set $D_{i}=\bigcup_{p \in \operatorname{crit}_{i}(f)} D_{i}(p)$. Then the inclusion $i: C_{*}^{\Delta}\left(\tilde{W}_{i-1} \cup \tilde{D}_{i}, \tilde{W}_{i-1}\right) \rightarrow$ $C_{*}^{\Delta}\left(\tilde{W}_{i}, \tilde{W}_{i-1}\right)$ is the inclusion of the core of the handles into the handles, hence a simple homotopy equivalence. Now $\varphi^{(k, k-1)} \circ i$ is a simple homotopy equivalence by Cohen $[1,18.3]$, since we can choose the lifts of $D_{i}$ so that the matrices representing $\varphi^{(k, k-1)} \circ i$ and the boundary operators have only integer values. Therefore $\varphi^{(k, k-1)}$ is a simple homotopy equivalence.

Now given another Morse function $g: W \rightarrow[0,1]$ and a transverse $g$-gradient $w$, let $\Phi: W \rightarrow W$ be isotopic to the identity such that $\Phi\left(W^{s}(q, v)\right)$ intersects $W^{u}(p, w)$ transversely for all critical points $q$ of $f$ and $p$ of $g$ with ind $q \leq$ ind $p$. The existence of such a $\Phi$ can be seen by adapting the proof of the existence of an adjusted triangulation. Let $\left(W_{i}(v)\right)_{i=-1}^{n}$ be a filtration by compact cobordisms to give the Morse-Smale complex with respect to $(f, v)$. By the transversality condition there is a $t>0$ such that $W_{i}^{t} \subset C_{i}(w)$ for all $i$ and hence a chain map

$$
\psi_{v, w}: C_{*}^{M S}\left(\tilde{W}, \tilde{M}_{0}, f, v\right) \rightarrow C_{*}^{M S}\left(\tilde{W}, \tilde{M}_{0}, g, w\right)
$$


induced by inclusion. For ind $q=$ ind $p$ we get that $\Phi\left(W^{s}(q, v)\right) \cap W^{u}(p, w)$ is a finite set and using the orientations and coorientations we get intersection numbers $[q: p] \in \mathbb{Z} G$ such that

$$
\psi_{v, w}(q)=\sum_{p \in \operatorname{crit}_{\text {ind } q}(f)}[q: p] p .
$$

A different choice of $\Phi$ will lead to a chain homotopic map, compare the proof of Lemma 2.4. The chain map $\psi_{v, w}$ is also a simple chain homotopy equivalence, more precisely we get

Proposition 2.6. Let $f_{0}, f_{1}, f_{2}: W \rightarrow[0,1]$ be Morse functions and let $v_{i}$ be $a$ transverse $f_{i}$-gradient for $i=0,1,2$. Then

(1) $\psi_{v_{0}, v_{1}} \circ \varphi_{v_{0}} \simeq \varphi_{v_{1}}$

(2) $\psi_{v_{1}, v_{2}} \circ \psi_{v_{0}, v_{1}} \simeq \psi_{v_{0}, v_{2}}$

Here ' $\simeq$ ' means 'chain homotopic'.

Proof. 1. Notice that if $\Phi$ is isotopic to the identity, then $f_{0} \circ \Phi$ is a Morse function and $d \Phi^{-1} \circ v_{0} \circ \Phi$ is an $f_{0} \circ \Phi$-gradient giving the same Morse-Smale complex as $\left(f_{0}, v_{0}\right)$. Therefore we assume that $\Phi$ is the identity. We can also assume the triangulation $\Delta$ is adjusted to both $v_{0}$ and $v_{1}$.

There is a $t>0$ such that $W_{i}^{t}\left(v_{0}\right) \subset C_{i}\left(v_{1}\right)$ for all $i$. Also there is an $s>0$ such that $\Theta_{s}^{v_{0}}\left(W^{(i)}\right) \subset W_{i}^{t}\left(v_{0}\right)$ for all $i$. Now $\Theta^{v_{0}}$ gives a homotopy between $\Theta_{s}^{v_{0}}$ and the identity. Modify this homotopy away from the endpoints to get a homotopy $h: W \times I \rightarrow W$ such that $h\left(W^{(i)} \times I\right) \subset C_{\tilde{i}+1}\left(v_{1}\right)$. Now define $H: C_{i}^{\Delta}\left(\tilde{W}, \tilde{M}_{0}\right) \rightarrow$ $C_{i+1}^{M S}\left(\tilde{W}, \tilde{M}_{0}, f_{1}, v_{1}\right)$ by sending $\sigma$ to $(-1)^{i} \tilde{h}_{*}[\tilde{\sigma} \times I] \in H_{i+1}\left(\tilde{C}_{i+1}\left(v_{1}\right), \tilde{C}_{i}\left(v_{1}\right)\right)$. Then

$$
\begin{aligned}
\partial H+H \partial(\sigma) & =\tilde{h}_{*}[\tilde{\sigma} \times 1]-\tilde{h}_{*}[\tilde{\sigma} \times 0] \\
& =\Theta_{s}^{v_{0}}[\tilde{\sigma}]-[\tilde{\sigma}] \\
& =\psi_{v_{0}, v_{1}} \circ \varphi_{v_{0}}(\sigma)-\varphi_{v_{1}}(\sigma)
\end{aligned}
$$

Notice that we evaluate in $H_{i}\left(\tilde{C}_{i}\left(v_{1}\right), \tilde{C}_{i-1}\left(v_{1}\right)\right)$. Now $\Theta_{s}^{v_{0}}[\tilde{\sigma}]$ represents $\varphi_{v_{0}}(\sigma)$ in $H_{i}\left(\tilde{W}_{i}^{t}\left(v_{0}\right), \tilde{W}_{i-1}^{t}\left(v_{0}\right)\right.$ and passing to $H_{i}\left(\tilde{C}_{i}\left(v_{1}\right), \tilde{C}_{i-1}\left(v_{1}\right)\right)$ is applying $\psi_{v_{0}, v_{1}}$.

To prove 2 . let us again assume that $\Phi$ can be chosen the identity, so that we have

$$
\begin{array}{lll}
W^{s}\left(q, v_{0}\right) & \pitchfork & W^{u}\left(p, v_{1}\right) \\
W^{s}\left(q, v_{0}\right) & \pitchfork & W^{u}\left(r, v_{2}\right) \\
W^{s}\left(p, v_{1}\right) & \pitchfork & W^{u}\left(r, v_{2}\right)
\end{array}
$$

for the relevant critical points.

Choose $t>0$ such that $W_{i}^{t}\left(v_{0}\right) \subset C_{i}\left(v_{1}\right) \cup C_{i}\left(v_{2}\right)$ and $W_{i}^{t}\left(v_{1}\right) \subset C_{i}\left(v_{2}\right)$ for all $i$. Since $W_{i}^{t}\left(v_{0}\right)$ is a compact subset of $C_{i}\left(v_{1}\right)$, there is an $s>0$ such that $\Theta_{s}^{v_{1}}\left(W_{i}^{t}\left(v_{0}\right)\right) \subset W_{i}^{t}\left(v_{1}\right)$ for all $i$. Let $h: W \times I \rightarrow W$ be a homotopy between the identity and $\Theta_{s}^{v_{1}}$ such that $h\left(W^{s}\left(p, v_{0}\right) \times I\right)$ intersects $W^{u}\left(r, v_{2}\right)$ transversely for ind $p \leq$ ind $r-1$. Using a lifting $\tilde{h}$ of $h$ we get a chain homotopy $H: C_{i}^{M S}\left(\tilde{W}, \tilde{M}_{0}, f_{0}, v_{0}\right)=H_{i}\left(W_{i}^{t}\left(v_{0}\right), W_{i-1}^{t}\left(v_{0}\right)\right) \rightarrow H_{i+1}\left(C_{i+1}\left(v_{2}\right), C_{i}\left(v_{2}\right)\right)=$ $C_{i+1}^{M S}\left(\tilde{W}, \tilde{M}_{0}, f_{2}, v_{2}\right)$ between $\psi_{v_{1}, v_{2}} \circ \psi_{v_{0}, v_{1}}$ and $\psi_{v_{0}, v_{2}}$ as in 1. 


\section{The Novikov Complex of a Circle valued Morse function}

In this section we look at a closed connected smooth manifold $M$ and smooth functions $f: M \rightarrow S^{1}$. If all critical points of $f$ are nondegenerate, we call $f$ a Morse function. Since locally we have a function to the reals we get an index for every critical point and there are only finitely many critical points. Let $\rho: \mathbb{R} \rightarrow S^{1}$ be the covering given by $\rho(x)=\exp (2 \pi i x)$. If the homomorphism of the fundamental group $f_{\#}: \pi_{1}(M) \rightarrow \pi_{1}\left(S^{1}\right)=\mathbb{Z}$ is trivial, $f$ can be lifted to a map to $\mathbb{R}$ and we are in the situation of Section 2. Therefore we assume that $f_{\#}$ is nontrivial. Then we can also assume that $f_{\#}$ is surjective since otherwise we can lift $f$ to a finite covering $S^{1} \rightarrow S^{1}$ such that the resulting map induces an epimorphism on fundamental group.

Let $\bar{\rho}: \bar{M} \rightarrow M$ be the connected infinite cyclic covering space corresponding to ker $f_{\#}$. Then $f \circ \bar{\rho}$ lifts to a map $\bar{f}: \bar{M} \rightarrow \mathbb{R}$ and we can assume that $0 \in \mathbb{R}$ is a regular value. Set $N=\bar{f}^{-1}(\{0\}), M_{N}=\bar{f}^{-1}([0,1])$ and $N^{\prime}=\bar{f}^{-1}(\{1\})$. Then $\left(M_{N} ; N, N^{\prime}\right)$ is a cobordism and $\bar{f}$ restricts to a Morse function on this cobordism. Let $\rho_{1}: \tilde{M} \rightarrow \bar{M}$ be a covering space of $\bar{M}$ with covering transformation group $H$ such that $\tilde{\rho}=\bar{\rho} \circ \rho_{1}: \tilde{M} \rightarrow M$ is a regular cover of $M$ with covering transformation group $G$. We then write $\tilde{f}=\bar{f} \circ \rho_{1}: \tilde{M} \rightarrow \mathbb{R}$ and $\tilde{X}=\rho_{1}^{-1}(X)$ for $X \subset \bar{M}$. Fix a covering transformation $t \in G$ such that $t\left(\tilde{N}^{\prime}\right)=\tilde{N}$. We have a short exact sequence

$$
1 \longrightarrow H \longrightarrow G \longrightarrow \mathbb{Z} \longrightarrow 1
$$

so that $G=H \times_{\theta} \mathbb{Z}$ is a semi direct product with $\theta: H \rightarrow H$ defined by $\theta(h)=$ $t^{-1} h t$. We can identify the group ring $\mathbb{Z} G$ with the $\theta$-twisted Laurent polynomial ring $\mathbb{Z} H_{\theta}\left[t, t^{-1}\right]$ where the elements are finite sums $\sum a_{j} t^{j}$ with $a_{j} \in \mathbb{Z} H$ and $a t=t \theta(a)$ for $a \in \mathbb{Z} H$. By abuse of notation we use $t$ both as an element of $G$ and as the indeterminate in the polynomial ring.

Let $\alpha$ be the standard closed 1 -form on $S^{1}$, i.e. such that $\rho^{*} \alpha=d x$.

Definition 3.1. A vector field $v$ is called an $f$-gradient, if it is dual to $f^{*} \alpha$ with respect to some Riemannian metric. It is called transverse, if $W^{s}(p, v)$ and $W^{u}(q, v)$ intersect transversely for all critical points of $f$ with ind $p \leq$ ind $q+1$.

Standard transversality arguments show that transverse $f$-gradients form a generic set among all $f$-gradients, see e.g. Pajitnov [15].

For every critical point $p$ of $f$ choose orientations, respectively coorientations, of $W^{s}(p, v)$, respectively $W^{u}(p, v)$, as in Section 2. Also choose a lift $\tilde{p} \in \tilde{M}$ for every critical point and lift the orientations as well. The lift of $v$ to $\bar{M}$ is denoted by $\bar{v}$ and the lift to $\tilde{M}$ by $\tilde{v}$.

Now define for $g_{1}, g_{2} \in G$ and two critical points $p, q$ with ind $p=$ ind $q+1$ $\left[g_{1} \tilde{p}: g_{2} \tilde{q}\right] \in \mathbb{Z}^{G}$ by $\left[g_{1} \tilde{p}: g_{2} \tilde{q}\right](g)=\sum \varepsilon(\tilde{\gamma})$, where the sum is taken over the trajectories of $-\tilde{v}$ from $g_{1} \tilde{p}$ to $g g_{2} \tilde{q}$.

Lemma 3.2. We have

(1) $\left[g_{1} \tilde{p}: g_{2} \tilde{q}\right]=g_{1}[\tilde{p}: \tilde{q}] g_{2}^{-1}$.

(2) $\left.\left[g_{1} \tilde{p}: g_{2} \tilde{q}\right]\right|_{H} \in \mathbb{Z} H$.

Proof. By definition $\left[g_{1} \tilde{p}: g_{2} \tilde{q}\right](g)=\left[g_{1} \tilde{p}: \tilde{q}\right]\left(g g_{2}\right)=\left[g_{1} \tilde{p}: \tilde{q}\right] \cdot g_{2}^{-1}(g)$. If $\tilde{\gamma}$ is a trajectory from $g_{1} \tilde{p}$ to $g g_{2} \tilde{q}$, then $g_{1}^{-1} \tilde{\gamma}$ is a trajectory from $\tilde{p}$ to $g_{1}^{-1} g g_{2} \tilde{q}$ with $\varepsilon\left(g_{1}^{-1} \tilde{\gamma}\right)=\varepsilon(\tilde{\gamma})$, so $\left[g_{1} \tilde{p}: g_{2} \tilde{q}\right](g)=\left[\tilde{p}: g_{2} \tilde{q}\right]\left(g_{1}^{-1} g\right)=g_{1} \cdot\left[\tilde{p}: g_{2} \tilde{q}\right](g)$. 
2. There exist integers $i<j$ such that $g_{1} \tilde{p}, g_{2} \tilde{q} \in \tilde{f}^{-1}([i, j])$. Then $\left.\left[g_{1} \tilde{p}: g_{2} q\right]\right|_{H}$ is the coefficient between $\rho_{1}\left(g_{1} \tilde{p}\right)$ and $\rho_{1}\left(g_{2} \tilde{q}\right)$ in the boundary of the Morse-Smale complex belonging to the cobordism $\bar{f}^{-1}([i, j])$.

Definition 3.3. The Novikov ring $\mathbb{Z} H_{\theta}((t))=\mathbb{Z} H_{\theta}[[t]]\left[t^{-1}\right]$ consists of formal power series $\sum_{j=-\infty}^{\infty} a_{j} t^{j}$ with $a_{j} \in \mathbb{Z} H$ and $\left\{j \leq 0 \mid a_{j} \neq 0\right\}$ is finite. Again $a t=t \theta(a)$ for $a \in \mathbb{Z} H$.

The Novikov complex of the circle valued Morse function $f: M \rightarrow S^{1}$ with transverse $f$-gradient $v$ is the finitely generated free $\mathbb{Z} H_{\theta}((t))$ complex $C_{*}(\tilde{M}, f, v)$ generated by the critical points of $f$ and graded by the index. The boundary is given by

$$
\partial_{i}(p)=\left.\sum_{q \in \mathrm{crit}_{i-1}(f)} \sum_{j=-\infty}^{\infty}\left[\tilde{p}: t^{j} \tilde{q}\right]\right|_{H} t^{j} q .
$$

Notice that for $\tilde{f}\left(t^{j} \tilde{q}\right)>\tilde{f}(\tilde{p})$ we get $\left.\left[\tilde{p}: t^{j} \tilde{q}\right]\right|_{H}=0$, so $\partial$ is a well defined module homomorphism. To see that $C_{*}(\tilde{M}, f, v)$ is indeed a chain complex we have to show that $\partial^{2}=0$. This is independent of the choice of liftings of critical points. For if we replace $\tilde{p}$ by $g \tilde{p}$ for some $g \in G$ to get a complex $C^{\prime}$, we can define $\varphi: C_{*}(\tilde{M}, f, v) \rightarrow C_{*}^{\prime}$ by $\varphi(q)=q$ for $q \neq p$ and $\varphi(p)=g^{-1} p$. Then $\partial^{\prime}=\varphi \circ \partial \circ \varphi^{-1}$. So choose the liftings of the critical points in $\tilde{M}_{N}$. For nonnegative integers $j$ let $M_{j}^{1}=\bar{f}^{-1}([-j, 1])$ and $M_{j}=\bar{f}^{-1}(\{-j\})$. Also let $\mathbb{Z} H_{\theta}[[t]]$ be the subring of $\mathbb{Z} H_{\theta}((t))$ consisting of formal power series $\sum_{j=0}^{\infty} a_{j} t^{j}$. That the Novikov complex is a chain complex now follows from the next Lemma.

Lemma 3.4. With the liftings chosen in $\tilde{M}_{N}$ we get that

$$
C_{*}(\tilde{M}, f, v)=\mathbb{Z} H_{\theta}((t)) \otimes_{\mathbb{Z} H_{\theta}[[t]]} \lim _{\longleftarrow} C_{*}^{M S}\left(\tilde{M}_{j}^{1}, \tilde{M}_{j}, \bar{f}|, \bar{v}|\right)
$$

where the inverse limit is a finitely generated free $\mathbb{Z} H_{\theta}[[t]]$ complex generated by the critical points of $f$.

Proof. For $i=0, \ldots, n$ let

$$
C_{i}(j)=\bar{f}^{-1}((-\infty, 1])-\bigcup_{k=0}^{j} \bigcup_{p, \text { ind } p \geq i+1} W^{u}\left(t^{k} p, \bar{v}\right) .
$$

Then $C_{i}^{M S}\left(\tilde{M}_{j}^{1}, \tilde{M}_{j}, \bar{f}|, \bar{v}|\right)=H_{i}\left(\tilde{C}_{i}(j), \tilde{C}_{i-1}(j)\right)$ is a free $\mathbb{Z} H$ module generated by the critical points of $\left.\bar{f}\right|_{M_{j}^{1}}$ having index $i$. The inclusion $M_{i}(j+1) \subset M_{i}(j)$ gives the inverse system of Morse-Smale complexes. It is now easy to see that the inverse limit is a free $\mathbb{Z} H_{\theta}[[t]]$ chain complex generated by the critical points of $f$ such that the boundary is given by (2), hence the result.

The inverse limit description is very useful in determining the chain homotopy type of the Novikov complex. Let $\Delta$ be a smooth triangulation of $M$, the term adjusted to $v$ carries over from the real valued case. The triangulation lifts to triangulations of $\bar{M}$ and $\tilde{M}$. By Section 2 we can find a triangulation adjusted to a compact cobordism $\bar{f}^{-1}([i, j])$ and get a certain openness and density result. Hence we can find a generic set of adjusted triangulations.

As in Section 2 we can now define intersection numbers $\left[g_{1} \tilde{\sigma}: g_{2} \tilde{p}\right] \in \mathbb{Z} G$ such that 
$\left[g_{1} \tilde{\sigma}: g_{2} \tilde{p}\right](g)$ is the signed number of points in $g_{1} \tilde{\sigma} \cap W^{u}\left(g g_{2} \tilde{p}, \tilde{v}\right)$. Lemma 3.2 carries over and we define $\varphi_{v}: C_{*}^{\Delta}(\tilde{M}) \rightarrow C_{*}(\tilde{M}, f, v)$ by

$$
\varphi_{v}(\sigma)=\left.\sum_{p, \text { ind } p=\operatorname{dim}} \sum_{j=-\infty}^{\infty}\left[\tilde{\sigma}: t^{j} \tilde{p}\right]\right|_{H} t^{j} p .
$$

Proposition 3.5. $\varphi_{v}$ is a chain homotopy equivalence.

Proof. Since $\varphi_{v}(\sigma)=\lim _{\longleftarrow} l_{*}: H_{i}(\tilde{\sigma}, \partial \tilde{\sigma}) \rightarrow H_{i}\left(\tilde{C}_{i}(j), \tilde{C}_{i-1}(j)\right)([\tilde{\sigma}])$, it is a chain map. Lemma 2.4 carries over. If the triangulation contains $N=f^{-1}(\{1\})$ as a subcomplex, we also get commutative diagrams

$$
\begin{array}{ccc}
C_{*}^{\Delta}\left(\tilde{M}_{j}^{1}, \tilde{M}_{j}\right) & \longleftarrow & C_{*}^{\Delta}\left(\tilde{M}_{j+1}^{1}, \tilde{M}_{j+1}\right) \\
\downarrow \varphi_{v}^{j} & \downarrow \varphi_{v}^{j+1} \\
C_{*}^{M S}\left(\tilde{M}_{j}^{1}, \tilde{M}_{j}, \bar{f}|, \bar{v}|\right) & \longleftarrow & C_{*}^{M S}\left(\tilde{M}_{j+1}^{1}, \tilde{M}_{j+1}, \bar{f}|, \bar{v}|\right)
\end{array}
$$

and $\varphi_{v}=\mathrm{id} \otimes \lim \varphi_{v}^{j}$. Since the $\varphi_{v}^{j}$ are chain homotopy equivalences by Theorem 2.5 , so is the inverse limit as a chain map between finitely generated free chain complexes inducing an isomorphism on homology. Therefore $\varphi_{v}$ is a chain homotopy equivalence.

Let us also define the chain maps $\psi_{v, w}: C_{*}(\tilde{M}, f, v) \rightarrow C_{*}(\tilde{M}, g, w)$ where $g$ : $M \rightarrow S^{-1}$ is another Morse function homotopic to $f$ and $w$ a transverse $g$-gradient. As in Section 2 we can assume, after possibly altering $g$ with a map isotopic to the identity on $M$, that $W^{s}(q, v)$ intersects $W^{u}(p, w)$ transversely for all critical points $q$ of $f$ and $p$ of $g$ with ind $q \leq$ ind $p$. For ind $q=\operatorname{ind} p$ and $g_{1}, g_{2} \in G$ define $\left[g_{1} \tilde{q}: g_{2} \tilde{p}\right] \in \mathbb{Z}^{G}$ such that $\left[g_{1} \tilde{q}: g_{2} \tilde{p}\right](g)$ is the signed number of points in $W^{s}\left(g_{1} \tilde{q}, \tilde{v}\right) \cap W^{u}\left(g g_{2} \tilde{p}, \tilde{w}\right)$. Then we define

$$
\psi_{v, w}(q)=\left.\sum_{p \in \operatorname{crit} \operatorname{tind}_{q}(g)} \sum_{j=-\infty}^{\infty}\left[\tilde{q}: t^{j} \tilde{p}\right]\right|_{H} t^{j} p .
$$

Proposition 3.6. Let $f_{i}: M \rightarrow S^{1}$ be homotopic Morse functions for and $v_{i}$ be transverse $f_{i}$-gradients for $i=0,1,2$. Then

(1) $\psi_{v_{0}, v_{1}} \circ \varphi_{v_{0}} \simeq \varphi_{v_{1}}$.

(2) $\psi_{v_{1}, v_{2}} \circ \psi_{v_{0}, v_{1}} \simeq \psi_{v_{0}, v_{2}}$.

Proof. We show 2. using Proposition 2.6, 1. will follow analogously. We assume the same transversality assumptions as in the proof of Proposition 2.6.

Let $\bar{f}_{i}: \bar{M} \rightarrow \mathbb{R}$ be liftings of the $f_{i}$ such that $0 \in \mathbb{R}$ is a regular value for all of them. There are integers $l, m$ such that $\bar{f}_{1}\left(\bar{f}_{0}^{-1}((-\infty, 0])\right) \subset(-\infty, l]$ and $\bar{f}_{2}\left(\bar{f}_{1}^{-1}((-\infty, l])\right) \subset(-\infty, m]$. Let $k_{0}=0, k_{1}=l$ and $k_{2}=m$. For $i=0,1,2$ define $M_{j}^{i}=\bar{f}_{i}^{-1}\left(\left[-j+k_{i}, 1+k_{i}\right]\right), N_{j}^{i}=\bar{f}_{i}^{-1}\left(\left\{-j+k_{i}\right\}\right)$ and $M_{\infty}^{i}=\bar{f}_{i}^{-1}\left(\left(-\infty, 1+k_{i}\right]\right)$. By using thin enough filtrations we get for $0 \leq i_{1}<i_{2} \leq 2$ chain maps $\psi_{i_{1} i_{2}}^{j}$ : $C_{*}^{M S}\left(\tilde{M}_{j}^{i_{1}}, \tilde{N}_{j}^{i_{1}}, \bar{f}_{i_{1}}\left|, \bar{v}_{i_{1}}\right|\right) \rightarrow C_{*}^{M S}\left(\tilde{M}_{j}^{i_{2}}, \tilde{N}_{j}^{i_{2}}, \bar{f}_{i_{2}}\left|, \bar{v}_{i_{2}}\right|\right)$ induced by inclusion as in the proof of Proposition 2.6. We also get a chain homotopy $H^{j}: \psi_{12}^{j} \circ \psi_{01}^{j} \simeq \psi_{02}^{j}$ which is induced by a homotopy $h^{j}: M_{\infty}^{0} \times I \rightarrow M_{\infty}^{2}$ between the inclusion $k_{02}: M_{\infty}^{0} \rightarrow M_{\infty}^{2}$ and $k_{12} \circ \Theta_{s_{j}}^{\bar{v}_{1}} \circ k_{01}$. Here $\Theta^{\overline{v_{1}}}$ is the flow of $-\overline{v_{1}}$ and the time $s_{j}$ is chosen so that the thin filtration of $M_{j}^{0}$ flows into the thin filtration of $M_{j}^{1}$. Also $h^{j}\left(W^{s}\left(\bar{p}, \bar{v}_{0}\right) \times I\right)$ 
intersects $W^{u}\left(\bar{r}, \bar{v}_{2}\right)$ transversely for ind $\bar{p} \leq$ ind $\bar{r}-1$ where $\bar{p} \in M_{j}^{0}$ and $\bar{r} \in M_{j}^{2}$. To define $H^{j+1}$ we need a new homotopy $h^{j+1}$. To get this use $h^{j}$ and flow a little longer along the flow of $-\bar{v}_{1}$ until the thin filtration of $M_{j+1}^{0}$ includes into the thin filtration of $M_{j+1}^{1}$. Then we change this homotopy to get a new homotopy $h^{j+1}$ such that $h^{j+1}\left(W^{s}\left(\bar{p}, \bar{v}_{0}\right) \times I\right)$ intersects $W^{u}\left(\bar{r}, \bar{v}_{2}\right)$ transversely for ind $\bar{p} \leq$ ind $\bar{r}-1$ where $\bar{p} \in M_{j+1}^{0}$ and $\bar{r} \in M_{j+1}^{2}$. Since this was already true for $\bar{p} \in M_{j}^{0}$ and $\bar{r} \in M_{j}^{2}$ we can make the changes so that the diagram

$$
\begin{array}{ccc}
C_{*}^{M S}\left(\tilde{M}_{j}^{0}, \tilde{N}_{j}^{0}, \bar{f}_{0}\left|, \bar{v}_{0}\right|\right) & \longleftarrow & C_{*}^{M S}\left(\tilde{M}_{j+1}^{0}, \tilde{N}_{j+1}^{0}, \bar{f}_{0}\left|, \bar{v}_{0}\right|\right) \\
\downarrow H^{j} & & \downarrow H^{j+1} \\
C_{*}^{M S}\left(\tilde{M}_{j}^{2}, \tilde{N}_{j}^{2}, \bar{f}_{2}\left|, \bar{v}_{2}\right|\right) & \longleftarrow & C_{*}^{M S}\left(\tilde{M}_{j+1}^{2}, \tilde{N}_{j+1}^{2}, \bar{f}_{2}\left|, \bar{v}_{2}\right|\right)
\end{array}
$$

commutes. The inverse limit of $H^{j}$ is a chain homotopy between $\lim \psi_{12}^{j} \circ \psi_{01}^{j}$ and $\longleftarrow \psi_{02}^{j}$. But $\psi_{v_{i_{1}}, v_{i_{2}}}=$ id $\otimes \lim \psi_{i_{1} i_{2}}^{j}$, if the liftings of the critical points of $f_{i}$ are chosen in $\tilde{f}^{-1}\left(\left[k_{i}, k_{i}+1\right]\right)$. Since the $\psi_{v_{0}, v_{1}}$ behave well with respect to change of basis we get the result.

Remark 3.7. The chain homotopy $H$ constructed in the proof can be written as

$$
H(p)=\sum_{r \in \operatorname{crit}_{\text {ind }} p+1} \sum_{j=-\infty}^{\infty}\left[p: t^{j} r\right] t^{j} r .
$$

To get $\left[p: t^{j} r\right](h) \neq 0$, there exists a trajectory of $-\tilde{v}_{0}$ from $\tilde{p}$ to a point $\tilde{x}$, a trajectory of $-\tilde{v}_{1}$ from $\tilde{x}$ to a point $\tilde{y}$ and a trajectory of $-\tilde{v}_{2}$ from $\tilde{y}$ to $h t^{j} \tilde{r}$. This observation will become useful in Section 4 .

\section{The Novikov complex of a Morse Closed 1-Form}

Now we want to look at closed 1-forms on a closed connected smooth manifold $M$. A closed 1-form $\omega$ induces a homomorphism $\xi_{[\omega]}=\xi: \pi_{1}(M) \rightarrow \mathbb{R}$ by $\xi([\gamma])=\int_{\gamma} \omega$, where $\gamma$ is a smooth representative of $[\gamma] \in \pi_{1}(M)$. By de Rham's theorem all homomorphisms $\xi: \pi_{1}(M) \rightarrow \mathbb{R}$ arise in such a way.

A closed 1-form is locally exact and we say that $\omega$ is a Morse form, if locally the functions $f: U \rightarrow \mathbb{R}$ with $d f=\left.\omega\right|_{U}$ have nondegenerate critical points only. Again we get that a Morse form has only finitely many critical points, each with an index.

Since $\pi_{1}(M)$ is finitely presented, im $\xi$ is a finitely generated subgroup of $\mathbb{R}$, hence $\mathbb{Z}^{k}$ for some $k \geq 0$. If $k=0, \omega$ is the differential of a smooth function $f: M \rightarrow \mathbb{R}$ and we are in the situation of Section 2. If $k=1$ we call $\omega$ rational and if $k>1$ we call $\omega$ irrational.

There is a minimal regular covering space $\bar{\rho}: \bar{M} \rightarrow M$ such that $\bar{\rho}^{*} \omega=d \bar{f}$ for some $\bar{f}: \bar{M} \rightarrow \mathbb{R}$, namely the one corresponding to ker $\xi$. The group of covering transformations is $\mathbb{Z}^{k}$. If $k=1$ let $t \in \mathbb{Z}$ be the generator such that $b=\bar{f}(\bar{x})-$ $\bar{f}(t \bar{x})>0$ for all $\bar{x} \in \bar{M}$. Then we can define $f: M \rightarrow S^{1}$ by $f(x)=\exp (2 \pi i \bar{f}(\bar{x}) / b)$, where $\bar{x} \in \bar{M}$ is a lift of $x \in M$. Then $\omega=b f^{*} \alpha$ with $\alpha$ the standard volume form on $S^{1}$ and we see that rational Morse forms correspond to circle valued Morse functions. 
Definition 4.1. Let $\omega$ be a Morse form. A vector field $v$ is called an $\omega$-gradient, if it is dual to $\omega$ with respect to some Riemannian metric. It is called transverse, if $W^{s}(p, v)$ and $W^{u}(q, v)$ intersect transversely for all critical points of $\omega$ with ind $p \leq$ ind $q+1$.

The following is an easy lemma proven in [21].

Lemma 4.2. Let $\omega$ be a Morse form and $v$ a vector field. Then $v$ is an $\omega$-gradient if and only if

(1) For every critical point $p$ of $\omega$ there exists a neighborhood $U_{p}$ of $p$ and a Riemannian metric $g$ on $U_{p}$ such that $\omega_{x}(X)=g(X, v(x))$ for every $x \in U_{p}$ and $X \in T_{x} U_{p}$.

(2) If $\omega_{x} \neq 0$, then $\omega_{x}(v(x))>0$.

We want to define a Novikov complex for a pair $(\omega, v)$ but we need the right ring for this. So for a group $G$ and a homomorphism $\xi: G \rightarrow \mathbb{R}$ let

$$
\widehat{\mathbb{Z}}_{\xi}=\left\{\lambda \in \mathbb{Z}^{G} \mid \forall R \in \mathbb{R} \#\{g \in G \mid \lambda(g) \neq 0 \text { and } \xi(g) \geq R\}<\infty\right\} .
$$

Clearly $\mathbb{Z} G \subset \widehat{\mathbb{Z} G}_{\xi}$ and the multiplication of $\mathbb{Z} G$ extends to $\widehat{\mathbb{Z} G}_{\xi}$. Then $\widehat{\mathbb{Z} G}_{\xi}$ becomes a ring, the Novikov ring.

For a surjective homomorphism $\xi: G \rightarrow \mathbb{Z}$ we want to show that this coincides with the Novikov ring given in Section 3. Let $t \in G$ satisfy $\xi(t)=-1$ and let $H=\operatorname{ker} \xi$. Let $\theta: H \rightarrow H$ be $\theta(h)=t^{-1} h t$. For every $g \in G$ there is a unique $n_{g} \in \mathbb{Z}$ and $h_{g} \in H$ such that $g=h_{g} \cdot t^{n_{g}}$. So for $a \in \mathbb{Z} H$ and $g \in G$ define $a t^{j} \in \mathbb{Z} G$ by $a t^{j}(g)=a\left(h_{g}\right) \cdot t^{j}\left(t^{n_{g}}\right)$. It is easy to see that this induces a ring isomorphism between $\mathbb{Z} H_{\theta}((t))$ and $\widehat{\mathbb{Z} G}_{\xi}$.

Given a Morse form $\omega$ and a transverse $\omega$-gradient we now want to define the Novikov complex $C_{*}(\tilde{M}, \omega, v)$ as before. For this we need the intersection numbers $[\tilde{p}: \tilde{q}]$ to be elements of $\widehat{\mathbb{Z}}_{\xi}$. We achieve this by an approximation result.

Lemma 4.3. Let $\omega$ be a Morse form and $v$ an $\omega$-gradient. There is a rational Morse form $\omega^{\prime}$ such that $v$ is also an $\omega^{\prime}$-gradient, $\omega^{\prime}$ agrees with $\omega$ in a neighborhood of the critical points of $\omega$ and the homomorphism $\xi_{\left[\omega^{\prime}\right]}$ vanishes on $\operatorname{ker} \xi_{[\omega]}$.

Proof. Let $g_{1}, \ldots, g_{k} \in G$ be a minimal set of generators of $\operatorname{im} \xi_{[\omega]}$ and let $\omega_{i}$ be closed 1 -forms for $i=1, \ldots, k$ such that $\xi_{i}=\xi_{\left[\omega_{i}\right]}: G \rightarrow \mathbb{Z}$ satisfies $\xi_{i}\left(g_{j}\right)=\delta_{i j}$ and all $\xi_{i}$ vanish on $\operatorname{ker} \xi_{[\omega]}$. We can assume that the $\omega_{i}$ vanish in a neighborhood of the critical points of $\omega$. Let $\varepsilon \in \mathbb{R}^{k}$. For $\|\varepsilon\|$ small enough the closed 1-form $\omega_{\varepsilon}=\omega+\sum_{i=1}^{k} \varepsilon_{i} \omega_{i}$ will have the property that $v$ is an $\omega_{\varepsilon}$-gradient by Lemma 4.2 . Now choose the $\varepsilon_{i}$ so that $\xi_{\left[\omega_{\varepsilon}\right]}$ factors through $\mathbb{Q}$.

So if $\omega$ is a Morse form, $v$ a transverse $\omega$-gradient, $\tilde{\rho}: \tilde{M} \rightarrow M$ a regular covering space with covering transformation group $G$ factoring through $\bar{M}$ we can define a Novikov complex $C_{*}\left(\tilde{M}, \omega^{\prime}, v\right)$ over $\widehat{\mathbb{Z} G}_{\xi^{\prime}}$ using $\omega^{\prime}$ from Lemma 4.3 and writing $\xi^{\prime}=\xi_{\left[\omega^{\prime}\right]}$. The next lemma shows that this complex pulls back to $\widehat{\mathbb{Z} G}_{\xi^{\prime}} \cap \widehat{\mathbb{Z} G}_{\xi} \subset \mathbb{Z}^{G}$, a subring of both $\widehat{\mathbb{Z} G}_{\xi^{\prime}}$ and $\widehat{\mathbb{Z}}_{\xi}$.

Lemma 4.4. Let $\omega_{1}, \omega_{2}$ be Morse forms that agree near the common set of critical points with corresponding homomorphisms $\xi_{1} \xi_{2}: G \rightarrow \mathbb{R}$. Let $v$ be both an $\omega_{1}$ - and $\omega_{2}$-gradient. Then there exist constants $A, B \in \mathbb{R}$ with $A>0$ such that whenever 
there is $g \in G$ and a trajectory $\tilde{\gamma}$ of $-\tilde{v}$ between the critical points gq and $\tilde{p}$, then $\xi_{1}(g) \leq A \xi_{2}(g)+B$.

Proof. For every pair of critical points $p, q$ of $\omega_{i}$ we can choose a path $\tilde{\gamma}_{p q}$ in $\tilde{M}$ from $\tilde{p}$ to $\tilde{q}$. There is a $K>0$ such that $\left|\int_{\gamma_{p q}} \omega_{i}\right| \leq K$ for $i=1,2$ and all pairs of critical points. Since $\omega_{1}$ and $\omega_{2}$ agree near the critical points there exists a $C \in(0,1)$ such that $\omega_{1}(v(x)) \geq C \omega_{2}(v(x))$ for all $x \in M$ by compactness. Now let $g \in G$ be as in the statement. Then

$$
\begin{aligned}
\xi_{1}(g) & =\int_{\gamma_{q p}} \omega_{1}+\int_{\gamma} \omega_{1} \leq K-\int_{-\infty}^{\infty} \omega_{1}(v(\gamma(t))) d t \\
& \leq K-C \int_{-\infty}^{\infty} \omega_{2}(v(\gamma(t))) d t \\
& \leq K+C K+C \int_{\gamma_{q p}} \omega_{2}+C \int_{\gamma} \omega_{2}=K(1+C)+C \xi_{2}(g),
\end{aligned}
$$

which gives the result.

Corollary 4.5. Let $\omega$ be a Morse form, $v$ a transverse $\omega$-gradient and $\omega^{\prime}$ as in Lemma 4.3. Let $p, q$ be critical points of $\omega$ with ind $p=\operatorname{ind} q+1$. Then $[\tilde{p}: \tilde{q}] \in$ $\widehat{\mathbb{Z} G}_{\xi^{\prime}} \cap \widehat{\mathbb{Z} G}_{\xi}$.

Definition 4.6. The Novikov complex of the Morse form $\omega$ and the transverse $\omega$-gradient $v$ is the free $\widehat{\mathbb{Z}}_{\xi}$ complex $C_{*}(\tilde{M}, \omega, v)$ generated by the critical points of $\omega$ and graded by the index. The boundary is given by

$$
\partial_{i}(p)=\sum_{q \in \operatorname{crit}_{i-1} \omega}[\tilde{p}: \tilde{q}] q .
$$

Since $[\tilde{p}: \tilde{q}] \in \widehat{\mathbb{Z} G}_{\xi^{\prime}} \cap \widehat{\mathbb{Z} G}_{\xi}$, we can define a graded module $C_{*}$ over $\widehat{\mathbb{Z}}_{\xi^{\prime}} \cap \widehat{\mathbb{Z} G}_{\xi}$ and a homomorphism $\partial$ given by (3). Tensoring with $\widehat{\mathbb{Z} G}_{\xi^{\prime}}$ gives the Novikov complex of Section 3, but since $\widehat{\mathbb{Z} G}_{\xi^{\prime}} \cap \widehat{\mathbb{Z} G}_{\xi}$ includes into $\widehat{\mathbb{Z} G}_{\xi^{\prime}}, C_{*}$ is already a chain complex. Now $C_{*}(\tilde{M}, \omega, v)=\widehat{\mathbb{Z} G}_{\xi} \otimes_{\widehat{\mathbb{Z} G}_{\xi^{\prime}} \cap \widehat{\mathbb{Z} G}_{\xi}} C_{*}$.

To identify the chain homotopy type we need the chain homotopy equivalences $\varphi_{v}: \widehat{\mathbb{Z} G}_{\xi} \otimes_{\mathbb{Z} G} C_{*}^{\Delta}(\tilde{M}) \rightarrow C_{*}(\tilde{M}, \omega, v)$ and $\psi_{v, w}: C_{*}\left(\tilde{M}, \omega_{1}, v\right) \rightarrow C_{*}\left(\tilde{M}, \omega_{2}, w\right)$ already known in the rational case. To get them as chain maps, we merely have to show that the intersection numbers $[\tilde{\sigma}: \tilde{p}]$ and $[\tilde{p}: \tilde{q}]$ as defined in Section 3 lie in $\widehat{\mathbb{Z} G}_{\xi^{\prime}} \cap \widehat{\mathbb{Z}}_{\xi}$ as well.

Proposition 4.7. Let $\omega_{0}, \omega_{1}, \omega_{2}$ be cohomologous Morse forms and for $i=0,1,2$ let $v_{i}$ be a transverse $\omega_{i}$-gradient. Then

(1) $\psi_{v_{0}, v_{1}} \circ \varphi_{v_{0}} \simeq \varphi_{v_{1}}$.

(2) $\psi_{v_{1}, v_{2}} \circ \psi_{v_{0}, v_{1}} \simeq \psi_{v_{0}, v_{2}}$.

Proof. Recall the proof of Lemma 4.3. By choosing the closed 1-forms to vanish in a neighborhood of all critical points of $\omega_{0}, \omega_{1}, \omega_{2}$ we can find rational approximations $\omega_{i}^{\prime}$ for $\omega_{i}$ such that $\omega_{0}^{\prime}, \omega_{1}^{\prime}$ and $\omega_{2}^{\prime}$ are cohomologous. In fact we get $\omega_{i}-\omega_{j}=\omega_{i}^{\prime}-\omega_{j}^{\prime}$. With Proposition 3.6 we get the $\varphi_{v_{i}}, \psi_{v_{i}, v_{j}}$ and the chain homotopies over $\widehat{\mathbb{Z} G}_{\xi^{\prime}}$. It remains to show that all the coefficients lie in $\widehat{\mathbb{Z G}}_{\xi^{\prime}} \cap \widehat{\mathbb{Z G}}_{\xi}$. This requires an argument similar to Lemma 4.4 and we give it for the chain homotopy $H: \psi_{v_{1}, v_{2}} \circ \psi_{v_{0}, v_{1}} \simeq$ 
$\psi_{v_{0}, v_{2}}$.

In Remark 3.7 we pointed out that a nonzero coefficient $[\tilde{p}: \tilde{r}]$ gives the existence of a trajectory $\tilde{\gamma}_{0}$ of $-\tilde{v}_{0}$ from $\tilde{p}$ to $\tilde{x} \in \tilde{M}$, a trajectory $\tilde{\gamma}_{1}$ of $-\tilde{v}_{1}$ from $\tilde{x}$ to $\tilde{y} \in \tilde{M}$ and a trajectory of $-\tilde{v}_{2}$ from $\tilde{y}$ to $g \tilde{r}$. As in Lemma 4.4 choose $K>0$ and paths $\tilde{\gamma}_{r p}$ between critical points $\tilde{r}$ of $\omega_{2}$ and $\tilde{p}$ of $\omega_{0}$ such that $\left|\int_{\gamma_{r p}} \omega_{i}\right| \leq K$. As in Lemma 4.4 there is a $C \in(0,1)$ with $\omega_{i}\left(v_{i}(x)\right) \geq C \omega_{i}^{\prime}\left(v_{i}(x)\right)$ for all $i=0,1,2$ and $x \in M$. Let $d f_{01}=\omega_{0}-\omega_{1}$ and $d f_{02}=\omega_{0}-\omega_{2}$. Then

$$
\begin{aligned}
\xi(g) & =\int_{\gamma_{r p}} \omega_{0}+\int_{\gamma_{0}} \omega_{0}+\int_{\gamma_{1}} \omega_{0}+\int_{\gamma_{2}} \omega_{0} \\
& =\int_{\gamma_{r p}} \omega_{0}+\int_{\gamma_{0}} \omega_{0}+\int_{\gamma_{1}} \omega_{1}+\int_{\gamma_{2}} \omega_{2}+\int_{\gamma_{1}} d f_{01}+\int_{\gamma_{2}} d f_{02} \\
& \leq K(1+C)+\int_{\gamma_{r p}} \omega_{0}^{\prime}+C \int_{\gamma_{0}} \omega_{0}^{\prime}+C \int_{\gamma_{1}} \omega_{1}^{\prime}+C \int_{\gamma_{2}} \omega_{2}^{\prime}+\int_{\gamma_{1}} d f_{01}+\int_{\gamma_{2}} d f_{02} \\
& =K(1+C)+(1-C)\left(\int_{\gamma_{1}} d f_{01}+\int_{\gamma_{2}} d f_{02}\right)+C \xi^{\prime}(g) .
\end{aligned}
$$

Notice that $\int_{\gamma_{i}} d f_{0 i}$ is bounded by $\max \left\{f_{0 i}(x)-f_{0 i}(y) \mid x, y \in M\right\}$. Therefore $[\tilde{p}$ : $\tilde{r}] \in \widehat{\mathbb{Z} G}_{\xi^{\prime}} \cap \widehat{\mathbb{Z} G}_{\xi}$ and the result follows.

Theorem 4.8. Let $\omega_{1}, \omega_{2}$ be cohomologous Morse forms, $v$ a transverse $\omega_{1}$-gradient and $w$ a transverse $\omega_{2}$-gradient. Then $\psi_{v, w}: C_{*}\left(\tilde{M}, \omega_{1}, v\right) \rightarrow C_{*}\left(\tilde{M}, \omega_{2}, w\right)$ and $\varphi_{v}: \widehat{\mathbb{Z} G}_{\xi} \otimes_{\mathbb{Z} G} C_{*}^{\Delta}(\tilde{M}) \rightarrow C_{*}\left(\omega_{1}, v\right)$ are chain homotopy equivalences.

Proof. That $\psi_{v, w}$ is a chain homotopy equivalence follows directly from Proposition 4.7.2 since $\psi_{v, v}=$ id by definition. By Proposition 4.7.1 it now suffices to show that $\varphi_{u}$ is a chain homotopy equivalence for a particular $\omega_{3}$ with transverse $\omega_{3}$-gradient $u$. By a nice trick of Latour [8] there is a Morse form $\omega_{3}$ cohomologous to $\omega_{1}$ and a transverse $\omega_{3}$-gradient $u$ such that $u$ is also a gradient of a Morse function $f: M \rightarrow \mathbb{R}$. Namely choose a Morse function $f: M \rightarrow \mathbb{R}$ and a transverse $f$ gradient $u$ and change $\omega_{1}$ to $\omega^{\prime}$ in a contractible neighborhood of every critical point of $f$ such that $\omega^{\prime}$ is constant 0 near the critical points of $f$. This is possible since $\omega_{1}$ is locally exact. Now for $C>0$ large enough $\omega_{3}=\omega^{\prime}+C \cdot d f$ has $u$ as a gradient. Then $C_{*}\left(\tilde{M}, \omega_{3}, u\right)=\widehat{\mathbb{Z} G}_{\xi} \otimes_{\mathbb{Z} G} C_{*}(\tilde{M}, f, u)$ and $\varphi_{u}=\mathrm{id} \otimes \varphi_{u}^{M S}$. Since $\varphi_{u}^{M S}$ is a chain homotopy equivalence, so is $\varphi_{u}$.

\section{Continuation}

Another way to describe a chain homotopy equivalence is the principle of continuation coming out of Floer theory, see also Schwarz [24] or Poźniak [19]. To describe this we will follow the exposition of Poźniak [19, §2.6].

Let $\omega_{0}$ and $\omega_{1}$ be cohomologous Morse forms and $v_{i}$ be a transverse $\omega_{i}$-gradient for $i=0,1$. We have $\omega_{0}-\omega_{1}=d f$ for some $f: M \rightarrow \mathbb{R}$. Define a Morse form $\Omega$ on $M \times[0,1]$ as follows. Let $v:[0,1] \rightarrow[0,1]$ be a smooth function constant 0 near 0 and constant 1 near 1 . For $C>0$ let

$$
\Omega=\omega_{0}-C \sin \pi t d t+d(f \cdot v)=\omega_{0}-C \sin \pi t d t+d f \cdot v(t)+f \cdot v^{\prime}(t) d t .
$$

We can choose the $C>0$ so large that the $d t$ summand only vanishes for $t=$ 0,1 . At $t=0$ we get $\omega=\omega_{0}$ and at $t=1$ we get $\Omega=\omega_{1}$. In particular the 
critical points of $\Omega$ are $(p, 0)$ with $p \in \operatorname{crit} \omega_{0}$ and $(q, 1)$ with $q \in \operatorname{crit} \omega_{1}$. Also ind $(p, 0)=\operatorname{ind} p+1$ and ind $(q, 1)=$ ind $q$. Now let $v$ be a transverse $\Omega$-gradient that agrees with $v_{i}$ on $M \times\{i\}$ for $i=0,1$. Then we can form the Novikov complex $C_{*}=C_{*}(\tilde{M} \times[0,1], \Omega, v)$ as before. To see that this is in fact a chain complex one can extend $\Omega$ to a Morse form on $M \times[0,2] /(x, 0)=(x, 2)=M \times S^{1}$ and get $C_{*}$ to be a certain restriction of the corresponding Novikov complex, see Poźniak [19] for more details. Now it is clear that $C_{i}=C_{i}\left(\tilde{M}, \omega_{1}, v_{1}\right) \oplus C_{i-1}\left(\tilde{M}, \omega_{0}, v_{0}\right)$ and $C_{*}\left(\tilde{M}, \omega_{1}, v_{1}\right)$ is a subcomplex. Therefore $C_{*}$ is the mapping cone of a chain map $c_{v_{0}, v_{1}}: C_{*}\left(\tilde{M}, \omega_{0}, v_{0}\right) \rightarrow C_{*}\left(\tilde{M}, \omega_{1}, v_{1}\right)$, the continuation map. It is obtained by counting the signed flowlines from $(p, 0)$ to $(q, 1)$. We show that $c_{v_{0}, v_{1}}$ is chain homotopic to $\psi_{v_{0}, v_{1}}$.

Let $p_{0}: C_{*} \rightarrow C_{*-1}\left(\tilde{M}, \omega_{0}, v_{0}\right)$ and $p_{1}: C_{*} \rightarrow C_{*}\left(\tilde{M}, \omega_{1}, v_{1}\right)$ be the projections. Notice that $p_{1}$ is not a chain map, in fact $p_{1} \partial_{i}=\partial_{i} p_{1}+(-1)^{i-1} c_{v_{0}, v_{1}} \circ p_{0}$.

Proposition 5.1. Let $\Delta$ be a smooth triangulation of $M$. Then $c_{v_{0}, v_{1}} \circ \varphi_{v_{0}} \simeq \varphi_{v_{1}}$.

Proof. We can assume that $\Delta$ is both adjusted to $v_{0}$ and $v_{1}$. We get a $C W$ structure $\Delta \times I$ of $M \times[0,1]$ by looking at the natural product structure. We want $\Delta \times I$ adjusted to $v$. Notice that for a critical point $(q, 1)$ of $\Omega$ the unstable manifold $W^{u}((q, 1), v)$ has one more dimension that $W^{u}\left(q, v_{1}\right)$ while $W^{u}((p, 0), v)=$ $W^{u}\left(p, v_{0}\right)$. So the simplices $\sigma \times\{1\}$ already intersect the $W^{u}((q, 1), v)$ transversely if $\sigma$ intersects $W^{u}\left(q, v_{1}\right)$ transversely. But the simplices $\sigma \times\{0\}$ do not intersect $W^{u}((p, 0), v)$ transversely even if $\sigma$ intersects $W^{u}\left(p, v_{0}\right)$ transversely. But we can extend $\Omega$ and $v$ into a collar of $M \times\{0\}$ and push the triangulation of $M \times\{0\}$ into that collar. Now we can adjust $\Delta \times I$ to $v$ such that the intersections of $W^{u}((p, 0), v)$ and $\sigma \times I$ give the same coefficient as the intersection of $\sigma$ with $W^{u}\left(p, v_{0}\right)$, i.e. we get $\varphi_{v_{0}}(\sigma)=p_{0} \varphi_{v}(\sigma \times I)$, where $\varphi_{v}: \widehat{\mathbb{Z} G}_{\xi} \otimes_{\mathbb{Z} G} C_{*}^{\Delta \times I}(\tilde{M} \times I) \rightarrow C_{*}$ is the resulting chain map. We can also assume that $p_{1} \varphi_{v}(\sigma \times\{1\})=\varphi_{v_{1}}(\sigma)$. Now define

$$
H_{i}: \widehat{\mathbb{Z}}_{\xi} \otimes_{\mathbb{Z} G} C_{i}(\tilde{M}) \rightarrow C_{i+1}\left(\tilde{M}, \omega_{1}, v_{1}\right)
$$

by $H_{i}(\sigma)=(-1)^{i} p_{1} \circ \varphi_{v}(\sigma \times I)$. Then

$$
\begin{aligned}
\partial_{i+1} H_{i}+H_{i-1} \partial_{i}(\sigma)= & (-1)^{i} \partial_{i+1} p_{1} \varphi_{v}(\sigma \times I)+(-1)^{i-1} p_{1} \varphi_{v}(\partial \sigma \times I) \\
= & (-1)^{i} p_{1} \partial_{i+1} \varphi_{v}(\sigma \times I)-c_{v_{0}, v_{1}} p_{0} \varphi_{v}(\sigma \times I)+ \\
& +(-1)^{i-1} p_{1} \varphi_{v}(\partial \sigma \times I) \\
= & p_{1} \varphi_{v}(\sigma \times\{1\})-p_{1} \varphi_{v}(\sigma \times\{0\})-c_{v_{0}, v_{1}} \varphi_{v_{0}}(\sigma) \\
= & \varphi_{v_{1}}(\sigma)-c_{v_{0}, v_{1}} \varphi_{v_{1}}(\sigma) .
\end{aligned}
$$

Notice that $p_{1} \varphi_{v}(\sigma \times\{0\})=0$, since $W^{u}((q, 1), v) \cap M \times\{0\}=\emptyset$.

Corollary 5.2. Let $\omega_{0}$ and $\omega_{1}$ be cohomologous Morse forms and $v_{i}$ be a transverse $\omega_{i}$-gradient for $i=0,1$. Then $c_{v_{0}, v_{1}}$ is chain homotopic to $\psi_{v_{0}, v_{1}}$.

Proof. Using Proposition 4.7, Theorem 4.8 and Proposition 5.1 we get

$$
c_{v_{0}, v_{1}} \simeq \varphi_{v_{1}} \circ \varphi_{v_{0}}^{-1} \simeq \psi_{v_{0}, v_{1}}
$$

hence the result. 


\section{The Simple homotopy type of the Novikov COMPleX}

In the exact case Theorem 2.5 already states that $\varphi_{v}$ is a simple chain homotopy equivalence, by Proposition 2.6.1 the same is true for $\psi_{v, w}$. In the nonexact case the situation gets a little bit more complicated. First we need to decide what we mean by a simple chain homotopy equivalence.

If $G$ is a group and $\xi: G \rightarrow \mathbb{R}$ a homomorphism, let $a \in \widehat{\mathbb{Z} G}_{\xi}$ satisfy $a(g)=0$ for $g \in G$ with $\xi(g) \geq 0$. Then $1-a \in \widehat{\mathbb{Z} G}_{\xi}$ is a unit with inverse $\sum_{k=0}^{\infty} a^{k} \in \widehat{\mathbb{Z} G}_{\xi}$. We call such units and units of the form $\pm g$ with $g \in G$ trivial units.

Definition 6.1. Let $G$ be a group and $\xi: G \rightarrow \mathbb{R}$ a homomorphism.

(1) Let $U$ be the subgroup of $K_{1}\left(\widehat{\mathbb{Z} G}_{\xi}\right)$ generated by the trivial units. Then $\mathrm{Wh}(G ; \xi):=K_{1}\left(\widehat{\mathbb{Z} G}_{\xi}\right) / U$

(2) A chain homotopy equivalence $\varphi: C_{*} \rightarrow D_{*}$ between finitely generated free based $\widehat{\mathbb{Z} G}_{\xi}$ complexes $C_{*}$ and $D_{*}$ is called simple, if $\tau(\varphi)=0 \in \mathrm{Wh}(G ; \xi)$.

In the rational case Pajitnov and Ranicki [18] obtain a decomposition result for $K_{1}\left(\widehat{\mathbb{Z}}_{\xi}\right)$. In particular the natural map $i_{*}: \mathrm{Wh}(G) \rightarrow \mathrm{Wh}(G ; \xi)$ is surjective then. Pajitnov [12] in the circle valued case and Latour [8] in general already showed that there is a simple chain homotopy equivalence between $C_{*}(\omega, v)$ and $\widehat{\mathbb{Z} G}_{\xi} \otimes_{\mathbb{Z} G} C_{*}^{\Delta}(\tilde{M})$ and the purpose of this section is to show that $\varphi_{v}$ is such an equivalence.

Theorem 6.2. Let $\omega_{1}, \omega_{2}$ be cohomologous Morse forms, $v$ a transverse $\omega_{1}$-gradient and $w$ a transverse $\omega_{2}$-gradient. Then $\tau\left(\varphi_{v}\right)=\tau\left(\psi_{v, w}\right)=\tau\left(c_{v, w}\right)=0 \in \operatorname{Wh}(G ; \xi)$, i.e. all these chain homotopy equivalences are simple.

Proof. We show that $\tau\left(\psi_{v, w}\right)=0$. By Corollary 5.2 we then get $\tau\left(c_{v, w}\right)=0$, since chain homotopic equivalences have the same torsion, see e.g. Ranicki [20, Prop.1.2]. To get the result for $\tau\left(\varphi_{v}\right)$ notice that by Proposition 4.7.1 it is enough to show $\tau\left(\varphi_{u}\right)=0$ for a particular $u$. Recall Latour's trick in the proof of Theorem 4.8: we get $\tau\left(\varphi_{u}\right)=i_{*} \tau\left(\varphi_{u}^{M S}\right)$ for the $u$ constructed there. Here $i_{*}: \operatorname{Wh}(G) \rightarrow \operatorname{Wh}(G ; \xi)$ is the natural map and $\tau\left(\varphi_{u}^{M S}\right)=0$ by Theorem 2.5. Hence we get $\tau\left(\varphi_{v}\right)=0$.

Since $\omega_{1}$ and $\omega_{2}$ are cohomologous, there is $f: M \rightarrow \mathbb{R}$ such that $\omega_{2}=\omega_{1}+d f$. Let $v:[0,1] \rightarrow[0,1]$ be a smooth function which is constant 0 near 0 and constant 1 near 1. Let $H^{\prime \prime}: M \times I \rightarrow T^{*} M$, where $T^{*} M$ is the cotangent bundle, be defined by $H^{\prime \prime}(x, t)=\left(\omega_{1}\right)_{x}+v(t) \cdot d f_{x}$. Then for every $t \in[0,1] H_{t}^{\prime \prime}=H^{\prime \prime}(\cdot, t)$ is cohomologous to $\omega_{1}$ with $H_{0}^{\prime \prime}=\omega_{1}$ and $H_{1}^{\prime \prime}=\omega_{2}$. Now adapt Milnor $[9, \S 2]$ to change $H^{\prime \prime}$ to $H^{\prime}: M \times I \rightarrow T^{*} M$ such that $H^{\prime}$ intersects the zero section transversely. Note that $[9, \S 2]$ improves a smooth function locally so it carries over to the closed 1-form case and also to a parametrized version. We do not need to change $H^{\prime \prime}$ near $M \times\{0,1\}$ so we can assume that $H_{0}^{\prime}=\omega_{1}, H_{1}^{\prime}=\omega_{2}$ and $H_{t}^{\prime}$ is cohomologous to $\omega_{1}$ for all $t \in[0,1]$.

Now $H^{\prime}$ intersects the zero section in finitely many intervals and circles. By using a small isotopy of $M \times I$ we can further arrange that the resulting $H: M \times I \rightarrow T^{*} M$ has the following properties:

(1) For all $t \in[0,1] H_{t}$ is cohomologous to $\omega_{1}$ and has only finitely many critical points.

(2) Every $H_{t}$ has at most one degenerate critical point.

(3) Fore only finitely many values of $t H_{t}$ has a degenerate critical point. 
(4) If $H_{t}$ has a degenerate critical point there is an $\varepsilon_{t}>0$ and a $\sigma_{t} \in\{-1,1\}$ such that $H_{t+\sigma_{t} \varepsilon}$ has exactly one more critical point than $H_{t}$ and $H_{t-\sigma_{t} \varepsilon}$ has exactly one less critical point for all $0<\varepsilon<\varepsilon_{t}$.

So let $t \in(0,1)$ be such that $H_{t}$ has a degenerate critical point. Denote this point by $(p, t) \in M \times[0,1]$. Let $U$ be a neighborhood of $(p, t)$ diffeomorphic to $\mathbb{R}^{n} \times(t-\varepsilon, t+\varepsilon)$ such that $(t-\varepsilon, t+\varepsilon) \subset[0,1]$ and $\varepsilon<\varepsilon_{t}$. Choose an $H_{t}$-gradient $v_{t}$ such that for all nondegenerate critical points the stable and unstable manifolds intersect transversely. By choosing $0<\delta<\varepsilon$ small enough we can find transverse $H_{t \pm \delta}$-gradients $v_{ \pm \delta}$ close to $v_{t}$. Without loss of generality we can assume that $H_{t+\delta}$ has two critical points in $\mathbb{R}^{n} \times\{t+\delta\} \subset U$. Let $U_{t}$ be the neighborhood of $p$ such that $U_{t} \times\{t\}=U \cap M \times\{t\}$ and let $h_{t}: U_{t} \rightarrow \mathbb{R}$ be such that $d h_{t}=\left.H_{t}\right|_{U_{t}}$. We can change $h_{t}$ slightly near $p$ to remove the critical point. Hence there is a contractible cylinder neighborhood $C$ of $p$. But we can also alter $h_{t}$ slightly near $p$ to introduce the two critical points of $H_{t+\delta}$ and for $\delta>0$ small enough we stay inside $C$. Then $C$ has a handlebody decomposition with two critical points. Hence the two critical points have adjacent index and the algebraic coefficient is \pm 1 .

Let $\left\{p_{1}, \ldots, p_{l}\right\}$ be the set of nondegenerate critical points of $H_{t}$. The closed 1-form $H_{t}$ pulls back to an exact form $d h$ with $h: \tilde{M} \rightarrow \mathbb{R}$. Choose liftings $\tilde{p}_{i} \in \tilde{M}$ of the critical points. If $\omega_{1}$ is irrational, we can choose them in $h^{-1}(J)$ where $J$ is an arbitrarily small interval. If $\omega_{1}$ is rational, we choose them in $h^{-1}([0, r])$, where $r>0$ is the positive generator of $\operatorname{im} \xi$.

Let $\left\{p_{1}^{-}, \ldots, p_{l}^{-}\right\}$be the set of critical points of $H_{t-\delta}$ and $\left\{p_{1}^{+}, \ldots, p_{l}^{+}, p_{l+1}^{+}, p_{l+2}^{+}\right\}$ be the set of critical points of $H_{t+\delta}$ such that $p_{i}^{ \pm}$corresponds to $p_{i}$ for $i=1, \ldots, l$. By choosing $\delta>0$ small enough we can make the $p_{i}^{ \pm}$arbitrarily close to $p_{i}$. Thus choose the liftings of $p_{i}^{ \pm}$close to the $\tilde{p}_{i}$.

Let us write $C_{*}\left(H_{t-\delta}, v_{-\delta}\right)=C_{*}^{-}$and $C_{*}\left(H_{t+\delta}, v_{+\delta}\right)=C_{*}^{+} \oplus D_{*}$, where $D_{*}$ is generated by the two extra critical points. Write the boundary of $C_{*}\left(H_{t+\delta}, v_{+\delta}\right)$ in this decomposition as $\left(\begin{array}{cc}\partial^{+} & c \\ 0 & \partial^{D}\end{array}\right)$. Now look at the matrix of $\psi=\psi_{v_{-\delta}, v_{+\delta}}$. By the definition of $\psi$ using the intersections of stable and unstable manifolds and the fact that the critical points $p_{i}^{+}$and $p_{i}^{-}$together with their stable and unstable manifolds are close to each other, we get the following description. In the irrational case it looks like $\psi=\left(\begin{array}{c}I-A \\ \psi_{D}\end{array}\right)$ with $A_{i j}(g)=0$ for $\xi(g) \geq 0$. In the rational case we can assume that $h\left(\tilde{p}_{i}\right) \leq h\left(\tilde{p}_{j}\right)$ for $i \leq j$ and the matrix looks like $\psi=\left(\begin{array}{c}I-A^{\prime}-O \\ \psi_{D}\end{array}\right)$ with $A_{i j}^{\prime}(g)=0$ for $\xi(g) \geq 0$ and $O$ a lower triangular and hence nilpotent matrix. Then $I+O+O^{2}+\ldots$ is elementary and $(I-A-O)\left(I+O+O^{2}+\ldots\right)=I-A$ with $A$ as before. Write $\psi_{C}=I-A$.

By choosing the liftings of $p_{l+1}^{+}$and $p_{l+2}^{+}$appropriately we get for the coefficient in the boundary of $D_{*}\left[\tilde{p}_{l+2}^{+}: \tilde{p}_{l+1}^{+}\right]=1-a$ with $a(g)=0$ for $\xi(g) \geq 0$.

We now have a short exact sequence of free $\widehat{\mathbb{Z} G}_{\xi}$ chain complexes

$$
0 \longrightarrow C_{*}^{-} \stackrel{\psi}{\longrightarrow} C_{*}^{+} \oplus D_{*} \longrightarrow \hat{D}_{*} \longrightarrow 0
$$

where $\hat{D}_{*}$ is the cokernel of $\psi$ and has rank 2. By Ranicki [20, Prop.1.8] the boundary of $\hat{D}_{*}$ is $\partial=\partial^{D}+\psi_{D} \psi_{C}^{-1} c$. 
We claim that if $\delta>0$ is small enough, then $\partial=1-b$ with $b(g)=0$ for $\xi(g) \geq 0$. Note that $\partial^{D}$ is already of that form. Now $c$ counts trajectories from $\tilde{p}_{l+2}^{+}$to translates of $\tilde{p}_{j}^{+}$and $\psi_{D}$ counts broken trajectories from $\tilde{p}_{j}^{-}$to translates of $\tilde{p}_{l+1}^{+}$. If $\delta>0$ is small enough $\tilde{p}_{l+2}^{+}$and $\tilde{p}_{l+1}^{+}$are so close that $\left(\psi_{D} \psi_{C}^{-1} c\right)(g) \neq 0$ implies $\xi(g)<0$.

By Ranicki [20, Prop.1.7] we now get that the projection $p: \mathcal{C}(\psi) \rightarrow \hat{D}_{*}$ from the mapping cone of $\psi$ to the cokernel is a chain homotopy equivalence with torsion

$$
\tau(p)=\sum_{i=0}^{\infty}(-1)^{i+1} \tau\left(\psi_{C}\right),
$$

so $\mathcal{C}(\psi)$ is simple homotopy equivalent to $\hat{D}_{*}$. But $\hat{D}_{*}$ has trivial torsion by the claim above. Therefore $\psi$ is a simple chain homotopy equivalence.

Now if there are no degenerate critical points between $H_{t-\delta}$ and $H_{t+\delta}$ the same argument, but easier since $\hat{D}_{*}=0$, shows that $\psi$ is a simple isomorphism. By Proposition 4.7 we get that $\psi_{v, w}$ is a simple chain homotopy equivalence.

Remark 6.3. In the rational case there is a direct proof that $\varphi_{v}$ is a simple chain homotopy equivalence using the inverse limit description. See [23, Rm.A.5] for details. That proof is much simpler, but it does not give the irrational case.

That the Novikov complex $C_{*}(\omega, v)$ is simple chain homotopy equivalent to $\widehat{\mathbb{Z}}_{\xi} \otimes_{\mathbb{Z} G}$ $C_{*}^{\Delta}(\tilde{M})$ is important for finding a minimal number of critical points for a Morse form within a cohomology class, see Latour [8] or [23]. Other applications of torsion are discussed in the next section.

\section{The ClOsED ORBIT STRUCTURE OF THE GRADIENT}

The torsions $\tau\left(\varphi_{v}\right)$ and $\tau\left(\psi_{v, w}\right)$ are already well defined in $K_{1}\left(\widehat{\mathbb{Z} G}_{\xi}\right) /\langle\tau( \pm g)| g \in$ $G\rangle$. If we denote by $W$ the subgroup of $K_{1}\left(\widehat{\mathbb{Z} G}_{\xi}\right) /\langle\tau( \pm g) \mid g \in G\rangle$ generated by the trivial units, we get $\tau\left(\varphi_{v}\right), \tau\left(\psi_{v, w}\right) \in W$ by Theorem 6.2. In this group the torsion $\tau\left(\varphi_{v}\right)$ does not depend on the triangulation of $M$, but it does depend on $v$. In fact it contains information about the closed orbit structure of $-v$. To make this more clear let us define a zeta function of $-v$. Let $\gamma \in H_{1}(M)$ be a nonzero homology class and let $C_{\gamma} \subset M \times(0, \infty)$ be the set of closed orbits of $-v$ belonging to $\gamma$. By [22, Lm.5.7] and Fuller [4, Th.3] $C_{\gamma}$ is an isolated compact set of closed orbits and hence its Fuller index $i\left(C_{\gamma}\right) \in \mathbb{Q}$, see Fuller [4], is defined. Note that $C_{\gamma} \neq \emptyset$ implies that $\xi(\gamma)<0$, since $\omega_{x}(v(x))<0$ for $\omega_{x} \neq 0$. Now $\bar{\eta}(-v) \in \mathbb{Q}^{G_{a b}}$ defined by $\bar{\eta}(-v)(\gamma)=i\left(C_{\gamma}\right)$ satisfies $\bar{\eta}(-v)(\gamma)=0$ for $\xi(\gamma) \geq 0$ and $\bar{\eta}(-v) \in \widehat{\mathbb{Q} G_{a b}}$. Then we define the commutative zeta function $\bar{\zeta}(-v)=\exp (\bar{\eta}(-v))$, where $\exp (x)=\sum_{k=0}^{\infty} \frac{x^{k}}{k !}$. A priori $\bar{\zeta}(-v)$ is an element of $\widehat{\mathbb{Q} G_{a b}}$, but one can write down a product formula to see that $\bar{\zeta}(-v) \in \widehat{\mathbb{Z} G_{a b}}$. But this also follows from the following.

Theorem 7.1. Let $\omega$ be a Morse form on the closed connected smooth manifold $M$ and $v$ a transverse $\omega$-gradient. Let $\bar{M}$ be the universal abelian covering space. Then for the chain homotopy equivalence $\varphi_{v}: \widehat{\mathbb{Z} G_{a b}} \otimes_{\mathbb{Z} G_{a b}} C_{*}(\bar{M}, \omega, v)$ we have $\operatorname{det}\left(\tau\left(\varphi_{v}\right)\right)=\bar{\zeta}(-v)$.

Sketch of proof. Let us assume that $\omega=f^{*} \alpha$, where $f: M \rightarrow S^{1}$ is a Morse function. As in Section 3 we get the cobordism $\left(M_{N} ; N, N^{\prime}\right)$. Pajitnov [13] defines 
a "cellularity" condition on $f$-gradients $v$. Roughly this condition requires a Morse decomposition on $N$ which also gives a decomposition on $N^{\prime}$ such that points from the $i$-skeleton of $N^{\prime}$ either flow into critical points of $f$ with index $\leq i$ or into the $i$-skeleton of $N$ under the flow of $-\bar{v}$. Here $\bar{v}$ is the lift of $v$ to $M_{N}$. A dual condition holds for the flow of $\bar{v}$.

Then the flow of $-\bar{v}$ defines a partially defined function $-\vec{v}: N^{\prime} \rightarrow N$ which behaves cellular. Furthermore the critical points of $\left.\bar{f}\right|_{M_{N}}$ together with the decomposition of $N$ gives a decomposition of $M_{N}$ such that its chain complex calculates $H_{*}\left(M_{N}\right)$. Also the flow of $-\bar{v}$ defines a cellular map $-\vec{v}: N^{\prime} \rightarrow M_{N}$ such that on the chain level we get a gradient-like chain approximation $h: C_{*}\left(\tilde{N}^{\prime}\right) \rightarrow C_{*}\left(\tilde{M}_{N}\right)$, see Ranicki $[20, \S 6]$ for this terminology. Using this chain approximation Ranicki $[20, \S 6]$ defines a chain homotopy equivalence $p: \widehat{\mathbb{Z}}_{\xi} \otimes_{\mathbb{Z} G} C_{*}^{\Delta}(\tilde{M}) \rightarrow C_{*}(\tilde{M}, \omega, v)$ with torsion

$$
\tau(p)=\sum_{k=0}^{n-1}(-1)^{k+1} \tau\left(1-t h_{k}^{N}\right) \in K_{1}\left(\mathbb{Z} H_{\theta}((t))\right)=K_{1}\left(\widehat{\mathbb{Z} G_{\xi}}\right) .
$$

Here $h_{*}^{N}: C_{*}\left(\tilde{N}^{\prime}\right) \rightarrow C_{*}(\tilde{N})$ is determined by the partially defined function $-\vec{v}$ : $N^{\prime} \rightarrow N$. In [21, Prop.4.1] a triangulation of $M$ is constructed such that $p=\varphi_{v}$. Therefore $\tau\left(\varphi_{v}\right)$ is given in terms of (4) as well. After passing to $\widehat{\mathbb{Z} G_{a b}}$ we get $\operatorname{det} \tau\left(\varphi_{v}\right)=\prod_{k=0}^{n-1} \operatorname{det}\left(1-t h_{k}^{N}\right)^{(-1)^{k+1}}$.

It remains to compare this to $\bar{\zeta}(-v)$. Note that closed orbits of $-v$ correspond to fixed points of iterates of $t^{-1} \circ-\vec{v}: N^{\prime} \rightarrow N^{\prime}$, where $t^{-1}: \bar{M} \rightarrow \bar{M}$ is the covering transformation sending $N$ to $N^{\prime}$. It is shown in Pajitnov [14, §8] that the fixed point information contained in $\operatorname{det} \tau\left(\varphi_{v}\right)$ matches the closed orbit information of the zeta function. This proves the theorem for cellular gradients. Now Pajitnov [13] shows such vector fields are $C^{0}$-generic. By showing that the torsion and the zeta function depend continuously on $v$ the theorem follows for general $v$. This is done in $[22, \S 8-\S 10]$.

In the case where $\omega$ is irrational the theorem can be shown by approximating $\omega$ with a rational Morse form, compare [21].

Notice that the torsion in (4) is over $\widehat{\mathbb{Z}}_{\xi}$, but the zeta function is defined in $\widehat{\mathbb{Z} G_{a b}}$. Since closed orbits do not give a well defined element of $G=\pi_{1}(M)$, we cannot define it in $\widehat{\mathbb{Z}}_{\xi}$. But the conjugacy class of a closed orbit in $G$ is well defined. Let $\Gamma$ be the set of conjugacy classes of $G$ and for $\gamma \in \Gamma$ let $C_{\gamma}$ be the set of closed orbits of $-v$ belonging to $\gamma$. Then $i\left(C_{\gamma}\right) \in \mathbb{Q}$ is well defined and we can define $\eta(-v) \in \widehat{\mathbb{Q} \Gamma}_{\xi}$ as before. Since $\widehat{\mathbb{Q} \Gamma}_{\xi}$ is only an abelian group, we cannot take the exponential of $\eta(-v)$.

A different approach to define a noncommutative zeta function was suggested by Geoghegan and Nicas [5] using Hochschild homology. If $\Phi: M \times \mathbb{R} \rightarrow M$ is the flow of $-v$, define for every positive integer $n$ a homotopy $F_{n}: M \times[0, n] \rightarrow M$ by restricting $\Phi$. For such an $F_{n}$ Geoghegan and Nicas [5] define the one-parameter trace $R\left(F_{n}\right) \in H H_{1}(\mathbb{Z} G)$. Now

$$
H H_{1}(\mathbb{Z} G) \cong \bigoplus_{\gamma \in \Gamma} C(g(\gamma))_{a b}
$$

where $g(\gamma) \in G$ represents the conjugacy class $\gamma$ and $C(g(\gamma))$ is the centralizer of $g(\gamma)$. 
A nonzero term in $C(g(\gamma))_{a b}$ detects a closed orbit which represents the conjugacy class $\gamma$. The following can be found in [22]. It is possible to complete $H H_{1}(\mathbb{Z} G)$ to $\widehat{H H}_{1}(\mathbb{Z} G)_{\xi}$ such that the sequence $R\left(F_{n}\right)$ converges in $\widehat{H H}_{1}(\mathbb{Z} G)_{\xi}$. Then the noncommutative zeta function is defined as $\zeta(-v)=\lim _{n \rightarrow \infty} R\left(F_{n}\right) \in \widehat{H H}_{1}(\mathbb{Z} G)_{\xi}$. Furthermore there is a natural homomorphism $l: \widehat{H H}_{1}(\mathbb{Z} G)_{\xi} \rightarrow \widehat{\mathbb{R}}_{\xi}$ such that $l(\zeta(-v))=\eta(-v)$.

To pass from $K$-theory to Hochschild homology we use the Dennis trace $D T$ : $K_{1}\left(\widehat{\mathbb{Z} G}_{\xi}\right) \rightarrow H H_{1}\left(\widehat{\mathbb{Z} G}_{\xi}\right)$. There is a natural homomorphism $\theta: H H_{1}\left(\widehat{\mathbb{Z} G}_{\xi}\right) \rightarrow$ $\widehat{H H}_{1}(\mathbb{Z} G)_{\xi}$. Now $\theta \circ D T$ does not vanish on $\operatorname{im}( \pm G) \subset K_{1}\left(\widehat{\mathbb{Z} G}_{\xi}\right)$, but $\theta \circ D T(\tau( \pm g))$ $=[g] \in C\left(1_{G}\right)_{a b}=G_{a b}$ which is a direct summand of $\widehat{H H}_{1}(\mathbb{Z} G)_{\xi}$ which contains no information of $\zeta(-v)$. By projecting this factor away we get a natural homomorphism $\mathfrak{D T}: K_{1}\left(\widehat{\mathbb{Z}}_{\xi}\right) /\langle\tau( \pm g) \mid g \in G\rangle \rightarrow \widehat{H H}_{1}(\mathbb{Z} G)_{\xi}$ and the generalization of Theorem 7.1 reads

Theorem 7.2. Let $\omega$ be a Morse form on the closed connected smooth manifold $M$ and $v$ a transverse $\omega$-gradient. Then for the chain homotopy equivalence $\varphi_{v}$ : $\widehat{\mathbb{Z} G}_{\xi} \otimes_{\mathbb{Z} G} C_{*}(\tilde{M}, \omega, v)$ we have $\mathfrak{D T}\left(\tau\left(\varphi_{v}\right)\right)=\zeta(-v)$.

The proof is analogous to the proof of Theorem 7.1, in fact we only need to check that the fixed point information contained in $\mathfrak{D T}\left(\tau\left(\varphi_{v}\right)\right)$ detects the zeta function. This is similar to the commutative case, but more involved, see $[22, \S 7]$ for details.

It is worth pointing out that $l \circ \mathfrak{D T}$ has a logarithm property, i.e. for a trivial unit $1-a$ we have $l \circ \mathfrak{D T}(\tau(1-a))=-\sum_{n=1}^{\infty} \frac{\varepsilon\left(a^{n}\right)}{n}$, where $\varepsilon: \widehat{\mathbb{Z} G}_{\xi} \rightarrow \widehat{\mathbb{Z}}_{\xi}$ is augmentation. Denote $\mathfrak{L}=l \circ \mathfrak{D T}$, this homomorphism was obtained by Pajitnov [16] without using Hochschild homology.

Pajitnov [14] defined for cellular gradients of circle valued functions a natural chain homotopy equivalence $\vartheta_{v}: C(\tilde{M}, f, v) \rightarrow \widehat{\mathbb{Z} G}_{\xi} \otimes_{\mathbb{Z} G} C_{*}^{\Delta}(\tilde{M})$. This is done by including the Novikov complex into a complex $C_{*}^{\prime}$ which is simply isomorphic to $\widehat{\mathbb{Z} G}_{\xi} \otimes_{\mathbb{Z} G} C_{*}^{\Delta}(\tilde{M})$ as used by Ranicki, compare [14, §7.4]. Composing this with $p: \overline{\mathbb{Z} G}_{\xi} \otimes_{\mathbb{Z} G} C_{*}^{\Delta}(\tilde{M})$ gives the identity on the Novikov complex. Since both maps are chain homotopy equivalences, they are mutually inverses to each other.

Proposition 7.3. Let $f: M \rightarrow S^{1}$ be a Morse function and $v$ a transverse cellular $f$-gradient. Then $\varphi_{v}$ and $\vartheta_{v}$ are mutually inverse chain homotopy equivalences.

Proof. This follows since different smooth triangulations do not change the chain homotopy type of $\varphi_{v}$ and for a special triangulation we get $\varphi_{v}=p$, see the proof of Theorem 7.1.

Pajitnov [16] obtains

$$
\mathfrak{L}\left(-\tau\left(\vartheta_{v}\right)\right)=\eta(-v) .
$$

Of course the proof of Theorem 7.2 uses results from [16], but Proposition 7.3 now shows how (5) follows from Theorem 7.2.

Also Pajitnov [12] defines a chain homotopy equivalence $\vartheta_{v}^{\prime}: C_{*}(\tilde{M}, f, v) \rightarrow \widehat{\mathbb{Z G}}_{\xi}$ $\otimes_{\mathbb{Z} G} C_{*}^{\Delta}(\tilde{M})$ without the cellularity assumption. In [17] Pajitnov shows that $\vartheta_{v}^{\prime}$ and $\vartheta_{v}$ are chain homotopic for cellular $v$, even though only for the minimal cover $\bar{M}$ instead of the universal cover.

A similar result to Theorem 7.1 has been obtained by Hutchings and Lee [7] and 
Hutchings [6]. Both require certain acyclicity conditions on the Novikov complex so the torsion of $\varphi_{v}$ is just the difference of two torsions. In particular $\varphi_{v}$ is not really needed. Nonetheless [7] contains a version of $\varphi_{v}$, using singular chains instead of simplices. While the method of [7] is similar to the one described in the proof of Theorem 7.1, the method of Hutchings [6] is quite different. There the change in the torsion of the Novikov complex and the zeta function when passing from one Morse form with gradient to another is shown to be equal by using bifurcation analysis. In view of Proposition 4.7 he shows that $\tau\left(\psi_{v, w}\right)$ detects the difference in zeta functions.

Let us finish by giving an example of a gradient for which the noncommutative zeta function contains more information than the commutative version. For this we need the following theorem which is proven in [23].

Theorem 7.4. Let $G$ be a finitely presented group, $\xi: G \rightarrow \mathbb{Z}$ a homomorphism with finitely presented kernel, $b \in \widehat{\mathbb{Z} G}_{\xi}$ satisfy $b(g)=0$ for $\xi(g) \geq 0$ and $n \geq 1$. Then for any closed connected smooth manifold $M$ with $\pi_{1}(M)=G$ and $\operatorname{dim} M \geq$ 6 there is a Morse function $f: M \rightarrow S^{1}$ realizing $\xi$ on fundamental group, a transverse $f$-gradient $v$ and $a b^{\prime} \in \widehat{\mathbb{Z} G}_{\xi}$ with $b^{\prime}(g)=b(g)$ for $\xi(g) \geq-n$ such that $\tau\left(\varphi_{v}\right)=\tau\left(1-b^{\prime}\right) \in K_{1}\left(\widehat{\mathbb{Z}}_{\xi}\right) /\langle\tau( \pm g) \mid g \in G\rangle$.

Now let $N$ be a nontrivial finitely presented nonabelian group. Then the projection $\xi: G=N \times \mathbb{Z} \rightarrow \mathbb{Z}$ satisfies the conditions of Theorem 7.4. Let $n \in[N, N]-\left\{1_{N}\right\}$. Then $g=(n,-1)$ and $h=\left(1_{N},-1\right)$ are not conjugated in $G$, but project to the same element in $G_{a b}=H_{a b} \times \mathbb{Z}$. Look at $\tau=\tau\left((1-g)(1-h)^{-1}\right) \in W$. Then $\varepsilon(\tau)=0 \in K_{1}\left(\mathbb{Z} H_{a b}((t))\right)$. To see that $\mathfrak{D T}(\tau) \neq 0$ we have the next Lemma.

Lemma 7.5. Let $G$ be a group and $\xi: G \rightarrow \mathbb{R}$ a homomorphism. For $g \in G$ let $p_{g}: \widehat{H H}_{1}(\mathbb{Z} G)_{\xi} \rightarrow C(g)_{a b}$ be the projection. If $\xi(g)<0$, then $p_{g} \circ \mathfrak{D T}(\tau(1-g)) \neq 0$ and $p_{h} \circ \mathfrak{D} \mathfrak{T}(\tau(1-g))=0$ if $h$ is not conjugated to $g^{n}$ for any positive $n$.

Proof. By $[22, \S 4] \mathfrak{D T}(\tau(1-g))$ is represented by a 1 -chain $-\sum_{k=0}^{\infty} g^{k} \otimes g$ and $p_{g}\left(\left[g^{k} \otimes g\right]\right)=[g] \in C(g)_{a b}$ for $k=0$ and 0 for $k>0$. Also $p_{h}\left(\left[g^{k} \otimes g\right]\right)=0$ if $h$ is not conjugated to $g^{k+1}$. To see that $[g] \neq 0 \in C(g)_{a b}$ note that $\xi: G \rightarrow \mathbb{R}$ restricts to $\xi: C(g) \rightarrow \mathbb{R}$ which induces $\bar{\xi}: C(g)_{a b} \rightarrow \mathbb{R}$ and clearly $\bar{\xi}([g])=\xi(g)<0$ by assumption.

In our situation we get $p_{g}(\mathfrak{D T}(\tau))=[g]$ and $p_{h}(\mathfrak{D T}(\tau))=-[h]$. Now apply Theorem 7.4 to get a manifold $M$, a Morse function $f: M \rightarrow S^{1}$ and a transverse $f$-gradient $v$ such that $\tau\left(\varphi_{v}\right)=\tau-\tau(1-b)$ where $b(g)=0$ for $\xi(g) \geq-1$. Then $\zeta(-v)$ detects two closed orbits corresponding to $g$ and $h$ while $\bar{\zeta}(-v)$ does not detect any closed orbits corresponding to $-1 \in \mathbb{Z}=H_{1}(M)$.

\section{REFERENCES}

[1] M. Cohen, Introduction to simple homotopy theory, Graduate Texts in Mathematics, Vol. 10. Springer-Verlag, New York-Berlin, 1973.

[2] M. Farber, Morse-Novikov critical point theory, Cohn localization and Dirichlet units, Commun. Contemp. Math. 1 (1999), 467-495.

[3] M. Farber and A. Ranicki, The Morse-Novikov theory of circle-valued functions and noncommutative localization, Tr. Mat. Inst. Steklova 225 (1999) 381-388.

[4] F. Fuller, An index of fixed point type for periodic orbits, Amer. J. Math. 89 (1967), 133-148.

[5] R. Geoghegan and A. Nicas Trace and torsion in the theory of flows, Topology 33 (1994), 683-719. 
D. SCHÜTZ

[6] M. Hutchings, Reidemeister torsion in generalized Morse theory, Forum Math. 14 (2002), 209-244.

[7] M. Hutchings and Y-J. Lee, Circle-valued Morse theory, Reidemeister torsion, and SeibergWitten invariants of three manifolds, Topology 38 (1999), 861-888.

[8] F. Latour, Existence de 1-formes fermées non singulières dans une classe de cohomologie de de Rham, Publ. IHES No.80 (1994), 135-194.

[9] J. Milnor, Lectures on the h-cobordism theorem, Notes by L. Siebenmann and J. Sondow, Princeton University Press, Princeton, N.J. 1965.

[10] J. Munkres, Elementary differential topology, Annals of Mathematics Studies, No. 54 Princeton University Press, Princeton, N.J. 1963.

[11] S. Novikov, Multivalued functions and functionals. An analogue of the Morse theory, Dokl. Akad. Nauk SSSR 260 (1981), 31-35. English translation in Soviet Math. Dokl. 24 (1981), 222-226.

[12] A. Pazhitnov, On the Novikov complex for rational Morse forms, Ann. Fac. Sci. Toulouse 4 (1995), 297-338.

[13] A. Pazhitnov, The incidence coefficients in the Novikov complex are generically rational functions, St. Petersburg Math. J. 9 (1998), 969-1006.

[14] A. Pajitnov, Simple homotopy type of the Novikov complex and Lefschetz $\zeta$-functions of the gradient flow, Russ. Math. Surveys 54 (1999), 119-169.

[15] A. Pajitnov, $C^{0}$-generic properties of boundary operators in the Novikov complex, Pseudoperiodic topology, Amer. Math. Soc. Transl. Ser. 2, 197 (1999), 29-115.

[16] A. Pajitnov, Closed orbits of gradient flows and logarithms of non-abelian Witt vectors, K-theory 21 (2000), 301-324.

[17] A. Pajitnov, Counting closed orbits of gradients of circle-valued maps, to appear in St. Petersburg Math. J., available as math.DG/0104273.

[18] A. Pajitnov and A. Ranicki, The Whitehead group of the Novikov ring, K-Theory 21 (2000), 325-365.

[19] M. Poźniak, Floer homology, Novikov rings and clean intersections. Northern California Symplectic Geometry Seminar, Amer. Math. Soc. Transl. Ser. 2, 196, 1999, 119-181.

[20] A. Ranicki, The algebraic construction of the Novikov complex of a circle-valued Morse function, Math. Annalen 322 (2002), 745-785.

[21] D. Schütz, Gradient flows of closed 1-forms and their closed orbits, Forum Math. 14 (2002), 509-537.

[22] D. Schütz, One parameter fixed point theory and gradient flows of closed 1-forms, K-theory 25 (2002), 59-97.

[23] D. Schütz, Controlled connectivity of closed 1-forms, Algebr. Geom. Topol. 2 (2002), 171-217.

[24] M. Schwarz, Equivalences for Morse homology, Geometry and topology in dynamics (Winston-Salem, NC, 1998/San Antonio, TX, 1999), Contemp. Math. 246 (1999), 197-216.

Max-Planck-Institut für Mathematik, Vivatsgasse 7, D-53111 Bonn, Germany

E-mail address: schuetz@mpim-bonn.mpg.de 